\title{
Integrable domain walls in ABJM theory
}

\section{Charlotte Kristjansen, ${ }^{a}$ Dinh-Long $\mathbf{V u}^{b}$ and Konstantin Zarembo ${ }^{a, b, 1}$}

${ }^{a}$ Niels Bohr Institute, Copenhagen University, Blegdamsvej 17, 2100 Copenhagen, Denmark

${ }^{b}$ Nordita, KTH Royal Institute of Technology and Stockholm University, Hannes Alfvéns Väg 12, 11419 Stockholm, Sweden

E-mail: kristjan@nbi.dk, dinh-long.vu@su.se, zarembo@nordita.org

ABSTRACT: One-point functions of local operators are studied, at weak and strong coupling, for the ABJM theory in the presence of a $1 / 2$ BPS domain wall. In the underlying quantum spin chain the domain wall is represented by a boundary state which we show is integrable yielding a compact determinant formula for one-point functions of generic operators.

Keywords: AdS-CFT Correspondence, Bethe Ansatz, D-Branes

ArXiv EPrint: 2112.10438

\footnotetext{
${ }^{1}$ Also at ITEP, Moscow, Russia.
} 


\section{Contents}

1 Introduction 1

2 The field theory set-up 2

2.1 A classical solution of the BPS equations 2

2.2 Nahm's equations 4

3 The dual string configuration $\quad 5$

3.1 Choice of metric 6

$\begin{array}{ll}3.2 & \text { The probe brane embedding }\end{array}$

$\begin{array}{lll}3.3 & \text { Chiral operators at strong coupling } & 9\end{array}$

4 One-point functions at weak coupling $\quad 9$

$\begin{array}{ll}4.1 \text { Boundary state overlaps } & 9\end{array}$

$\begin{array}{lll}4.2 & \text { Selection rules } & 11\end{array}$

$\begin{array}{ll}\text { 4.3 Chiral primary operators at weak coupling } & 12\end{array}$

5 Excited states $\quad 14$

$\begin{array}{lll}5.1 & \mathrm{SU}(4) \text { sector } & 14\end{array}$

$\begin{array}{llr}5.2 & \text { Full spectrum } & 18\end{array}$

$\begin{array}{lll}5.2 .1 & \text { Fermionic dualities } & 18\end{array}$

$\begin{array}{lll}5.2 .2 & \operatorname{OSp}(6 \mid 4) \text { overlaps } & 19\end{array}$

6 One-point functions at strong coupling $\quad 21$

7 Conclusion and outlook $\quad 24$

\section{Introduction}

The study of domain wall set-ups featuring Nahm poles in $\mathcal{N}=4$ SYM has provided us with novel examples of integrable boundary states which, owing to the integrable $\operatorname{PSU}(2,2 \mid 4)$ super spin chain underlying the $A d S_{5} / C F T_{4}$ correspondence [1], have a discrete realization as matrix product states or valence bond states [2-4]. Overlaps between these integrable boundary states and Bethe eigenstates encode the one-point functions of the field theory in the presence of the domain wall. For the simplest1/2 BPS D3-D5 domain wall the overlaps are now known in a closed form at any loop order [5-7] and at least in a certain sub-sector non-perturbatively as well [7]. Prior to this, numerous partial results were obtained at fixed loop order and in particular sub-sectors and served as input for the bootstrap idea leading to the progress above $[2-4,8-10]$. These results were based on the powerful machinery of boundary integrability in quantum spin chains, and recent progress in this area [11] will be instrumental in our analysis as well. For a more involved non-supersymmetric D3-D7 set-up the overlaps are known at the leading loop order [12]. 
A fully non-perturbative analysis of the integrability of the boundary conditions would most likely require a treatment of the overlaps by means of the quantum spectral curve [13] the basics of which is encoded in the QQ-system for the integrable super spin chain [14]. An analysis of the overlap formulas of the D3-D5 domain wall model from the point of view of the QQ-system revealed that these formulas were singled out by exhibiting specific covariance properties under the fermionic duality transformations implied by the QQsystem [15]. This raised the hope that a covariance criterion could be used to constrain or even to fully determine yet unknown overlap formulas.

Another system where one could imagine closed overlap formulas to exist is a domain wall version of ABJM theory. First of all the $A d S_{4} / C F T_{3}$ system has a description as an integrable $\operatorname{OSp}(6 \mid 4)$ super spin chain $[16,17]$ and secondly integrable matrix product states were already discovered in the study of determinant operators in ABJM theory [18] as they were in $\mathcal{N}=4$ SYM theory [19].

In the present paper we point out that there exists a $1 / 2$ BPS domain wall version of ABJM theory, with a string theory dual taking the form of a D2-D4 probe brane system with flux, which shares many characteristics with the D3-D5 domain wall version of $\mathcal{N}=4 \mathrm{SYM}$ and for which the one-point functions can be found in closed form. The BPS conditions can again be expressed as a set of Nahm equations and the one-point functions can be calculated both at strong and at weak coupling (so far at the leading order). Furthermore, in both cases the probe D-brane configuration without flux corresponds to an integrable boundary condition according to an analysis carried out by Dekel and $\mathrm{Oz}[20]$. We determine the overlap formula of the ABJM domain wall model in the scalar sector by exploiting a newly derived result for overlaps in a class of bosonic spin chains [11] and use the requirement of covariance under fermionic dualities to uniquely fix the formula for the full ABJM theory. We also perform computations of one-point functions from the string theory perspective.

Our paper is organized as follows. We begin by presenting the domain wall version of ABJM theory in section 2 and in particular turning the BPS condition into Nahm equations for certain field combinations. Then we turn to describing the dual string theory set-up in section 3. The subsequent two sections are devoted to the calculation of one-point functions in the field theory language addressing protected states in section 4 and excited states in section 5. Section 6 concerns the calculation of one-point functions in the string theory language. Finally, section 7 contains our conclusion and outlook.

\section{The field theory set-up}

\subsection{A classical solution of the BPS equations}

The ABJM model [21] is a Chern-Simons-matter theory in three dimensions with $\mathrm{U}(N) \times$ $\mathrm{U}(N)$ gauge symmetry and the Lagrangian [22]

$$
\begin{aligned}
\mathcal{L}= & \frac{k}{4 \pi} \operatorname{tr}\left[\varepsilon^{\mu \nu \lambda}\left(A_{\mu} \partial_{\nu} A_{\lambda}+\frac{2}{3} A_{\mu} A_{\nu} A_{\lambda}-\hat{A}_{\mu} \partial_{\nu} \hat{A}_{\lambda}-\frac{2}{3} \hat{A}_{\mu} \hat{A}_{\nu} \hat{A}_{\lambda}\right)\right. \\
& +D_{\mu} Y_{A}^{\dagger} D^{\mu} Y^{A}+\frac{1}{12} Y^{A} Y_{A}^{\dagger} Y^{B} Y_{B}^{\dagger} Y^{C} Y_{C}^{\dagger}+\frac{1}{12} Y^{A} Y_{B}^{\dagger} Y^{B} Y_{C}^{\dagger} Y^{C} Y_{A}^{\dagger} \\
& \left.-\frac{1}{2} Y^{A} Y_{A}^{\dagger} Y^{B} Y_{C}^{\dagger} Y^{C} Y_{B}^{\dagger}+\frac{1}{3} Y^{A} Y_{B}^{\dagger} Y^{C} Y_{A}^{\dagger} Y^{B} Y_{C}^{\dagger}+\text { fermions }\right] .
\end{aligned}
$$


The scalars $Y^{A}$ belong to the bi-fundamental representation of the gauge group and transform as 4 of the SU(4) R-symmetry: $A=1, \ldots, 4$. At large $N$ and fixed 't Hooft coupling

$$
\lambda=\frac{N}{k},
$$

the model is dual to string theory on $A d S_{4} \times \mathbb{C} P^{3}$ with the dimensionless string tension $T \simeq \sqrt{\lambda / 2}[21]$.

The domain wall (at weak coupling) is described by a classical scalar-field profile that satisfies the equations of motions. More precisely, it must fulfill a set of simpler BPS equations, in order to preserve part of the supersymmetry. The energy of a BPS configuration takes the smallest possible value allowed by the boundary conditions. Once the energy density is represented as a total square plus a total derivative yielding a boundary term, the BPS condition requires the total square to vanish. The potential energy in (2.1) is not a total square in general, but becomes such when evaluated on a configuration with only two fields excited. We denote those $Y^{1}$ and $Y^{2}$, or collectively $Y^{\alpha}$.

This follows from an identity

$$
\begin{aligned}
\operatorname{tr} Y_{\alpha}^{\dagger} Y^{\beta} Y_{\gamma}^{\dagger} Y^{\alpha} Y_{\beta}^{\dagger} Y^{\gamma}= & 3 \operatorname{tr} Y_{\alpha}^{\dagger} Y^{\beta} Y_{\beta}^{\dagger} Y^{\alpha} Y_{\gamma}^{\dagger} Y^{\gamma}-\operatorname{tr} Y_{\alpha}^{\dagger} Y^{\beta} Y_{\beta}^{\dagger} Y^{\gamma} Y_{\gamma}^{\dagger} Y^{\alpha} \\
& -\operatorname{tr} Y_{\alpha}^{\dagger} Y^{\alpha} Y_{\beta}^{\dagger} Y^{\beta} Y_{\gamma}^{\dagger} Y^{\gamma},
\end{aligned}
$$

derived by contracting $\varepsilon^{\alpha \beta \gamma} \varepsilon_{\delta \varepsilon \omega}=0$ with $Y_{\alpha}^{\dagger} Y^{\delta} Y_{\beta}^{\dagger} Y^{\varepsilon} Y_{\gamma}^{\dagger} Y^{\omega}$, and thus is only valid for $\alpha, \beta, \ldots=1,2$. One of the tensor structures in (2.1) becomes redundant and the horribly looking energy functional collapses into a neat modulus-squared form:

$$
\begin{aligned}
E= & \frac{k}{4 \pi} \int d x \operatorname{tr}\left(\frac{d Y_{\alpha}^{\dagger}}{d x} \mp \frac{1}{2} Y_{\beta}^{\dagger} Y^{\beta} Y_{\alpha}^{\dagger} \pm \frac{1}{2} Y_{\alpha}^{\dagger} Y^{\beta} Y_{\beta}^{\dagger}\right) \\
& \times\left(\frac{d Y^{\alpha}}{d x} \mp \frac{1}{2} Y^{\alpha} Y_{\beta}^{\dagger} Y^{\beta} \pm \frac{1}{2} Y^{\beta} Y_{\beta}^{\dagger} Y^{\alpha}\right)+\text { total derivative }
\end{aligned}
$$

where $x \equiv x_{2}$ is the coordinate transverse to the domain wall. Either sign can be taken, corresponding to BPS and anti-BPS solutions. We take the upper sign for definiteness.

The BPS equations correspond to the absolute minimum of the energy in a given "topological sector", when the integrand is set to zero altogether:

$$
\frac{d Y^{\alpha}}{d x}=\frac{1}{2} Y^{\alpha} Y_{\beta}^{\dagger} Y^{\beta}-\frac{1}{2} Y^{\beta} Y_{\beta}^{\dagger} Y^{\alpha} .
$$

In [23] these equations were obtained by demanding the supersymmetry variation of the fermions to vanish. The solution will consequently host fermion zero modes and will automatically preserve half of the supersymmetry.

The solution found in [23] has a scale-invariant form

$$
Y^{\alpha}=\frac{S^{\alpha}}{\sqrt{x}}, \quad x>0
$$

where $S^{\alpha}$ are two rectangular matrices

$$
S_{i j}^{1}=\delta_{i, j-1} \sqrt{i}, \quad S_{i j}^{2}=\delta_{i j} \sqrt{q-i}, \quad i=1, \ldots, q-1 \quad j=1, \ldots, q .
$$


Assuming $q \leqslant N$ such a solution can be placed in the upper left corner of the $N \times N$ matrix fields $Y^{A}$ with $A=1,2$. The resulting configuration can be checked to solve the BPS equations. It breaks the gauge symmetry down to $\mathrm{U}(N-q+1) \times \mathrm{U}(N-q)$ for finite values of $x$ whereas asymptotically as $x \rightarrow+\infty$ the symmetry becomes $\mathrm{U}(N) \times \mathrm{U}(N)$. On the other side of the domain wall, $x<0$, the gauge symmetry is taken to be $\mathrm{U}(N-q+1) \times \mathrm{U}(N-q)$. Incidentally, the same matrices $S^{\alpha}$ describe the non-Abelian Coulomb branch of the massdeformed ABJM theory [24].

\subsection{Nahm's equations}

Before proceeding, we would like to make contact with Nahm's equations [25] that describe supersymmetric domain walls in $4 \mathrm{D}[26,27]$ and naturally arise in $\mathcal{N}=4$ super-YangMills theory with boundaries or defects [28]. Nahm's equations have a clear geometric interpretation in the large- $N$ limit encapsulating the spherical shape of the brane embedded in $S^{5}$ through the fuzzy, non-commutative geometry of the solution at large but finite $N$. We will argue that the BPS equation (2.5) is, in some sense, a square root of the Nahm's equation.

Consider, to this end, a composite field

$$
\Phi_{\beta}^{\alpha}=Y^{\alpha} Y_{\beta}^{\dagger}
$$

Assuming that $Y^{\alpha}$ satisfy the BPS condition (2.5), we can differentiate $\Phi_{\beta}^{\alpha}$ after $x$ to get a closed system of equations:

$$
\frac{d \Phi_{\beta}^{\alpha}}{d x}=\Phi_{\gamma}^{\alpha} \Phi_{\beta}^{\gamma}-\frac{1}{2}\left\{\Phi_{\gamma}^{\gamma}, \Phi_{\beta}^{\alpha}\right\}
$$

These equations can be further simplified by expanding the composite field in the $(\boldsymbol{\sigma}, \mathbb{1})$ basis:

$$
\Phi_{\beta}^{\alpha}=\Phi^{i} \sigma_{i \beta}^{\alpha}+\Phi \delta_{\beta}^{\alpha} .
$$

For the expansion coefficients we find, after simple algebra:

$$
\begin{aligned}
\frac{d \Phi^{i}}{d x} & =\frac{i}{2} \varepsilon^{i j k}\left[\Phi^{j}, \Phi^{k}\right], \\
\frac{d \Phi}{d x} & =\Phi^{i} \Phi^{i}-\Phi^{2} .
\end{aligned}
$$

The first equality is the Nahm equation.

The BPS domain wall (2.6) corresponds to the simplest Nahm-pole solution:

$$
\Phi^{i}=\frac{t^{i}}{x}
$$

where, in virtue of (2.11), $t^{i}$ must satisfy the $\mathfrak{s u}(2)$ commutation relations:

$$
\left[t^{i}, t^{j}\right]=i \varepsilon^{i j k} t^{k}
$$


The $(q-1) \times(q-1)$ matrices $t^{i}$ thus form a $(q-1)$-dimensional representation of $\mathfrak{s u}(2)$. The Casimir $\Phi^{i} \Phi^{i}$ determines the singlet component $\Phi$ through eq. (2.12):

$$
\Phi=\frac{q \mathbb{1}}{2 x} .
$$

The connection to the $\mathfrak{s u}(2)$ representation theory is quite fascinating. And it fits well with the dual supergravity description. The D4-brane representing the domain wall wraps a $\mathbb{C} P^{1}=S^{2}$ in $\mathbb{C} P^{3}$, and a large $\mathfrak{s u}(2)$ representation can be interpreted as a fuzzy twosphere that becomes smooth in the large- $q$ limit. The appearance of a fuzzy two-sphere in connection with the BPS equations of ABJM theory was also discussed in [29, 30].

The same chain of arguments applies to the dual bi-linear:

$$
\hat{\Phi}_{\alpha}{ }^{\beta}=Y_{\alpha}^{\dagger} Y^{\beta} \equiv \hat{\Phi}_{i} \sigma_{\alpha}^{i \beta}+\hat{\Phi} \delta_{\alpha}{ }^{\beta} .
$$

The triplet component again satisfies Nahm's equations and the singlet is determined by the Casimir $\hat{\Phi}_{i} \hat{\Phi}_{i}$ :

$$
\begin{aligned}
\frac{d \hat{\Phi}_{i}}{d x} & =-\frac{i}{2} \varepsilon_{i j k}\left[\hat{\Phi}_{j}, \hat{\Phi}_{k}\right], \\
\frac{d \hat{\Phi}}{d x} & =-\hat{\Phi}_{i} \hat{\Phi}_{i}+\hat{\Phi}^{2} .
\end{aligned}
$$

The Nahm-pole solution describing the domain wall is

$$
\begin{aligned}
\hat{\Phi}_{i} & =-\frac{\hat{t}_{i}}{x}, \quad\left[\hat{t}_{i}, \hat{t}_{j}\right]=i \varepsilon_{i j k} \hat{t}_{k}, \\
\hat{\Phi} & =\frac{(q-1) \mathbb{1}}{2 x} .
\end{aligned}
$$

The simplest domain wall solution has $q=2$. The bilinear (2.8) then is a $1 \times 1$ matrix (only its 11 component is non-zero), and since in the $1 \mathrm{~d}$ representation $t^{i}$ are trivial the bi-linear field takes a super-simple form:

$$
\Phi_{\beta}^{\alpha}=\frac{\delta_{\beta}^{\alpha}}{x} \quad(q=2) .
$$

The dual bilinear $(2.16)$ is a $2 \times 2$ matrix:

$$
\hat{\Phi}_{\alpha}^{\beta}=\frac{\mathbb{1} \delta_{\alpha}^{\beta}-\sigma_{3} \sigma_{i} \sigma_{3} \sigma_{\alpha}^{i \beta}}{2 x} \quad(q=2) .
$$

\section{The dual string configuration}

We expect the string theory configuration dual to the BPS solution of the previous section to be a D2-D4 probe brane system in type IIA superstring theory where the probe brane has geometry $A d S_{3} \times \mathbb{C} P^{1} \subset A d S_{4} \times \mathbb{C} P^{3}$ and carries $q$ units of world volume gauge field flux on the $\mathbb{C} P^{1}$. We can find such a probe brane embedding by extremizing the DBI plus WZ action for a D4-brane in the $A d S_{4} \times \mathbb{C} P^{3}$ background as outlined in [31]. The probe brane embedding involving the wrapping of a $\mathbb{C} P^{1} \subset \mathbb{C} P^{3}$ matches the $\mathrm{SU}(2) \times \mathrm{SU}(2) \times \mathrm{U}(1)$ symmetry of the vevs of the scalar fields of the Chern Simons field theory and, as we shall see, the additional flux accounts for the jump in the rank of the gauge group across the domain wall. 


\subsection{Choice of metric}

Let us start by choosing an appropriate parametrization of $S^{7}$. We follow reference [32] and introduce four complex variables as follows

$$
\begin{aligned}
Z^{1} & =\cos (\xi) \cos \left(\frac{\theta_{1}}{2}\right) e^{i\left(\chi_{1}+\phi_{1}\right) / 2}, & Z^{2} & =\cos (\xi) \sin \left(\frac{\theta_{1}}{2}\right) e^{i\left(\chi_{1}-\phi_{1}\right) / 2}, \\
Z^{3} & =\sin (\xi) \cos \left(\frac{\theta_{2}}{2}\right) e^{i\left(\chi_{2}+\phi_{2}\right) / 2}, & Z^{4} & =\sin (\xi) \sin \left(\frac{\theta_{2}}{2}\right) e^{i\left(\chi_{2}-\phi_{2}\right) / 2},
\end{aligned}
$$

where $\xi \in\left[0, \frac{\pi}{2}\left[\right.\right.$ and $\theta_{1}, \theta_{2} \in[0, \pi]$. Furthermore, $\phi_{1}, \phi_{2} \in[0,2 \pi]$ and $\chi_{1}, \chi_{2} \in[0,4 \pi[$. With this parametrization the metric of $S^{7}$ can be written as

$$
\begin{aligned}
d s_{S^{7}}^{2}= & d \xi^{2}+\frac{\cos ^{2} \xi}{4}\left[\left(d \chi_{1}+\cos \theta_{1} d \phi_{1}\right)^{2}+d \theta_{1}^{2}+\sin ^{2} \theta_{1} d \phi_{1}^{2}\right] \\
& +\frac{\sin ^{2} \xi}{4}\left[\left(d \chi_{2}+\cos \theta_{2} d \phi_{2}\right)^{2}+d \theta_{2}^{2}+\sin ^{2} \theta_{2} d \phi_{2}^{2}\right] .
\end{aligned}
$$

Next, we define new coordinates by

$$
\chi_{1}=2 y+\psi, \quad \chi_{2}=2 y-\psi,
$$

where $y \in[0,2 \pi], \psi \in[-2 \pi, 2 \pi]$. Then we can implement the quotient $S^{7} / \mathbb{Z}_{k}$ by making the identification

$$
y \sim y+\frac{2 \pi}{k} .
$$

We can now also rewrite the metric of $S^{7}$ as

$$
d s_{S^{7}}^{2}=d s_{\mathbb{C} P^{3}}^{2}+(d y+A)^{2},
$$

where

$$
A=\frac{1}{2}\left(\cos ^{2} \xi-\sin ^{2} \xi\right) d \psi+\frac{1}{2} \cos ^{2} \xi \cos \theta_{1} d \phi_{1}+\frac{1}{2} \sin ^{2} \xi \cos \theta_{2} d \phi_{2},
$$

and

$$
\begin{aligned}
d s_{\mathbb{C} P^{3}}^{2}= & d \xi^{2}+\cos ^{2} \xi \sin ^{2} \xi\left(d \psi+\frac{\cos \theta_{1}}{2} d \phi_{1}-\frac{\cos \theta_{2}}{2} d \phi_{2}\right)^{2} \\
& +\frac{1}{4} \cos ^{2} \xi\left(d \theta_{1}^{2}+\sin ^{2} \theta_{1} d \phi_{1}^{2}\right)+\frac{1}{4} \sin ^{2} \xi\left(d \theta_{2}^{2}+\sin ^{2} \theta_{2} d \phi_{2}^{2}\right) .
\end{aligned}
$$

For $A d S_{4}$ we can use the Poincaré metric

$$
d s_{A d S_{4}}^{2}=\frac{1}{z^{2}} d z^{2}+z^{2}\left(-d x_{0}^{2}+d x_{1}^{2}+d x_{2}^{2}\right),
$$

where the AdS boundary is at $z \rightarrow \infty$.

The relevant 10-dimensional background of type IIA string theory is described by the metric

$$
d s^{2}=\widetilde{R}^{2}\left(d s_{A d S_{4}}^{2}+4 d s_{\overparen{C} P^{3}}^{2}\right),
$$

where

$$
\frac{\widetilde{R}^{2}}{\alpha^{\prime}}=\pi \sqrt{\frac{2 N}{k}} .
$$


The background comes with a RR 2-form field as well as a RR 4-form field which are given by

$$
\begin{aligned}
F^{(2)}= & k\left(-\cos \xi \sin \xi d \xi \wedge\left(2 d \psi+\cos \theta_{1} d \phi_{1}-\cos \theta_{2} d \phi_{2}\right)\right. \\
& \left.-\frac{1}{2} \cos ^{2} \xi \sin \theta_{1} d \theta_{1} \wedge d \phi_{1}-\frac{1}{2} \sin ^{2} \xi \sin \theta_{2} d \theta_{2} \wedge d \phi_{2}\right), \\
F^{(4)}= & \frac{3 R^{3}}{8} \epsilon_{A d S_{4}},
\end{aligned}
$$

where $\epsilon_{A d S_{4}}$ is the volume form on $A d S_{4}$, and $\left(\frac{R}{l_{p}}\right)^{3}=4 k\left(\frac{\widetilde{R}}{\sqrt{\alpha^{\prime}}}\right)^{2}$.

\subsection{The probe brane embedding}

We are interested in a D4 probe with geometry $A d S_{3} \times \mathbb{C} P^{1}$ embedded in the IIA background. We make the ansatz that the probe brane is placed at $\xi=0$ (and $\theta_{2}, \phi_{2}, \psi$ constant) and we take its world volume coordinates to be $z, x_{0}, x_{1}, \theta_{1}, \phi_{1}$ while the last embedding coordinate $x_{2}$ is supposed to be non-constant but to depend only on $z$. The $\xi=0$ condition singles out a $\mathbb{C} P^{1} \subset \mathbb{C} P^{3}$ wrapped by the brane and parametrized by the coordinates $\theta_{1}, \phi_{1}$. The brane likewise wraps an $A d S_{3} \subset A d S_{4}$ parametrized by the coordinates $z, x_{0}, x_{1}$. Furthermore, we turn on a world-volume gauge field on the $\mathbb{C} P^{1}$ given by the 2 -form

$$
\mathcal{F}=\widetilde{R}^{2} Q \sin \theta_{1} d \theta_{1} \wedge d \phi_{1}
$$

As we shall see, the parameter $Q$ is related to the rank of the representation of the classical fields in the gauge theory, $q$. The probe Dp-brane action in general takes the form

$$
\begin{aligned}
I & =I_{\mathrm{DBI}}+I_{\mathrm{WZ}} \\
& =-T_{p} \int d^{p+1} \sigma e^{-\Phi} \sqrt{-\operatorname{det}(G+\mathcal{F})}+T_{p} \int d^{p+1} \sigma e^{\mathcal{F}} \wedge \sum_{m} \mathcal{P}\left[C_{m}\right],
\end{aligned}
$$

where $G$ is the induced metric on the brane, $\mathcal{F}$ is the world volume gauge field and the $C_{m}$ 's are the various RR background gauge field potentials where we have set the Kalb-Ramond field to zero. Furthermore $\mathcal{P}$ stands for the pull-back. In the present case the $C_{m}$ 's are $C_{1}$ and $C_{3}$, related to the field strengths via $F^{(4)}=d C_{3}$ and $F^{(2)}=d C_{1}$. In our case only the term with $C_{3}$ will be non-vanishing and thus the Wess-Zumino terms reads

$$
I_{\mathrm{WZ}}=T_{4} \int d^{5} \sigma \mathcal{F} \wedge \mathcal{P}\left[C_{3}\right] .
$$

Finding the induced metric $G$ is straightforward and we get

$$
-\operatorname{det}(G+\mathcal{F})=\widetilde{R}^{10} z^{2}\left(1+z^{4}\left(x_{2}^{\prime}(z)\right)^{2}\right) \sin ^{2} \theta_{1}\left(1+Q^{2}\right) .
$$

For $C_{3}$ we can take

$$
C_{3}=\frac{R^{3}}{8} z^{3} d x^{0} \wedge d x^{1} \wedge d x^{2}
$$

and we find

$$
I_{\mathrm{WZ}}=T_{4} \widetilde{R}^{4} \frac{k}{2} \int d^{5} \sigma\left(Q \sin \theta_{1} z^{3} x_{2}^{\prime}(z)\right)
$$


We now get for the total action

$$
\begin{aligned}
I_{\mathrm{DBI}+\mathrm{WZ}} & =T_{4} \frac{k}{2} \widetilde{R}^{4} \int_{-\infty}^{\infty} d x^{0} \int_{-\infty}^{\infty} d x^{1} \int_{0}^{\pi} d \theta_{1} \sin \theta_{1} \int_{0}^{2 \pi} d \phi_{1} \int_{0}^{\infty} d z \mathcal{I} \\
& =2 \pi \sqrt{\alpha^{\prime}} k T_{4} \widetilde{R}^{4} V \int_{0}^{\infty} d z \mathcal{I},
\end{aligned}
$$

where $V=\int d x^{0} d x^{1}$ and

$$
\mathcal{I}=\left[-z \sqrt{\left(1+z^{4}\left(x_{2}^{\prime}(z)\right)^{2}\right)\left(1+Q^{2}\right)}+Q z^{3} x_{2}^{\prime}(z)\right],
$$

and where we have made use of the relation

$$
e^{-\Phi}=\frac{k \sqrt{\alpha^{\prime}}}{2 \widetilde{R}}
$$

From this we get the following Euler Lagrange equation for $x_{2}(z)$

$$
\frac{\partial}{\partial z}\left\{\frac{z \sqrt{1+Q^{2}}}{\sqrt{1+z^{4}\left(x_{2}^{\prime}(z)\right)^{2}}} z^{4} x_{2}^{\prime}(z)-Q z^{3}\right\}=0,
$$

and we see that it has the solution

$$
x_{2}(z)=\frac{Q}{z}
$$

which is the solution we expect to be relevant for our analysis. The parameter $Q$ should be associated with the number, $q$, of D2 branes ending on the D4 brane and thus the jump in the rank of the gauge group across the defect in the field theory language. The relation can be found by interpreting the Wess-Zumino term of the D4 brane as the coupling of a number, $q$, of $\mathrm{D} 2$ branes to the background gauge field $C^{3}$ and leads to

$$
q=\frac{T_{4}}{T_{2}} \int_{S^{2}} \mathcal{F}=\frac{T_{4}}{T_{2}} 4 \pi \widetilde{R}^{2} Q=\frac{\widetilde{R}^{2}}{\pi \alpha^{\prime}} Q=\sqrt{\frac{2 N}{k}} Q,
$$

where we have made use of the relation

$$
T_{p}=(2 \pi)^{-p}\left(\alpha^{\prime}\right)^{-(p+1) / 2},
$$

as well as eq. (3.9). In particular we see that only if we take the parameter $Q$ finite will the probe brane have an angle with the $A d S_{4}$ boundary which is different from $\frac{\pi}{2}$. The probe brane configuration thus suggests the following double scaling limit

$$
\lambda \rightarrow \infty, \quad q \rightarrow \infty, \quad \frac{2 \lambda}{q^{2}}=Q^{-2} \quad \text { fixed }
$$

This situation is reminiscent of the situation in the $A d S_{5} / C F T_{4}$ correspondence where similar Karch-Randall probe brane set-ups of D3-D5 and D3-D7 type likewise suggested the introduction of a double scaling limit involving the 't Hooft coupling constant in combination with a certain representation label $[33,34]$. In the $A d S_{5} / C F T_{4}$ case this double scaling parameter allowed for a successful comparison of one-point functions of chiral primaries computed in gauge theory and in string theory to two leading orders both for a supersymmetric D3-D5 probe brane set-up [33, 35, 36] and two non-supersymmetric D3-D7 probe brane set-ups $[34,37,38]$. In the present case we shall see that a successful comparison is only possible if an additional large charge limit is imposed. 


\subsection{Chiral operators at strong coupling}

One-point functions of chiral primary operators in the presence of a Karch-Randall probe brane can be calculated through a fluctuation analysis of the supergravity background. The analysis will be carried out in section 6 . Only chiral primaries whose symmetries are compatible with the $\mathrm{SU}(2) \times \mathrm{SU}(2) \times \mathrm{U}(1)$ symmetry of the brane embedding will have non-vanishing one-point functions. These particular chiral primaries depend only on the angular variable $\xi$ and are solutions of the Laplace equation

$$
\nabla^{2} Y=\frac{1}{\sqrt{g}} \partial_{i} \sqrt{g} g^{i j} \partial_{j} Y=-E Y,
$$

which reduces to

$$
\nabla^{2} Y=\frac{1}{\sin ^{3} \xi \cos ^{3} \xi} \partial_{\xi} \sin ^{3} \xi \cos ^{3} \xi \partial_{\xi} Y=-E Y .
$$

The most general solution of this equation is a linear combination of a hypergeometric function and a Meijers G-function. In order for the solution to be non-singular at $\cos \xi=0$ one has to discard the Meijers G-function, and in order for the solution to be regular at $\cos \xi=1$ one needs

$$
E=2 \Delta(2 \Delta+6),
$$

where we recognize $\Delta$ as the conformal dimension of the corresponding operator. The solution more precisely reads

$$
Y_{\Delta}(\xi)=\mathcal{N}_{\Delta} \cdot{ }_{2} F_{1}\left[-\Delta, \Delta+3,2, \cos ^{2} \xi\right],
$$

where $\mathcal{N}_{\Delta}$ is a normalization factor.

\section{One-point functions at weak coupling}

In analogy with the situation in $\mathcal{N}=4 \mathrm{SYM}[2,3]$, tree level one-point functions in the scalar sector of ABJM theory can be expressed as overlaps between Bethe eigenstates and Matrix Product States. Furthermore, as the bond dimension of the Matrix Product State becomes equal to one, the Matrix Product State becomes a valence bond state. In the present section we expound these ideas and compute the one-point functions of protected scalar operators. The more intricate case of excited states is treated in the subsequent section.

\subsection{Boundary state overlaps}

The single-trace scalar operators of ABJM can be viewed as states in an alternating SU(4) spin chain:

$$
\mathcal{O}=\Psi_{A_{1} \ldots A_{2 L-1}}^{A_{2} \ldots A_{2 L}} \operatorname{tr} Y^{A_{1}} Y_{A_{2}}^{\dagger} \ldots Y^{A_{2 L-1}} Y_{A_{2 L}}^{\dagger} .
$$

The odd and even sites are occupied intermittently by $\mathbf{4}$ and $\overline{\mathbf{4}}$ of SU(4). The two-loop mixing matrix of scalar operators is identified with the spin-chain Hamiltonian [16]:

$$
H=\lambda^{2} \sum_{l=1}^{2 L}\left(1-P_{l, l+2}+\frac{1}{2} P_{l, l+2} K_{l, l+1}+\frac{1}{2} K_{l, l+1} P_{l, l+2}\right),
$$


where $K_{l m}$ and $P_{l m}$ are the standard trace and permutation operators acting on sites $l$ and $m$ :

$$
P_{A B}^{A^{\prime} B^{\prime}}=\delta_{B}^{A^{\prime}} \delta_{A}^{B^{\prime}}, \quad K_{B^{\prime}}^{A^{\prime}}{ }_{B}^{A}=\delta_{B^{\prime}}^{A^{\prime}} \delta_{B}^{A} .
$$

The permutation always acts on the same type of spins, while the trace mixes the two representations.

Traceless and symmetric tensors (symmetric in each set of indices) define chiral primary operators. At fixed length they belong to the same representation of $\mathfrak{s u}(4)$, the one with Dynkin labels $[L, 0, L]$. All chiral primaries have zero energy and form the ground state multiplet of the spin-chain Hamiltonian. One can declare the lowest-weight state

$$
\mathcal{O}_{\text {vac }}=\operatorname{tr}\left(Y^{1} Y_{2}^{\dagger}\right)^{L},
$$

the "true" vacuum and generate all other members of the multiplet by inserting zeromomentum, zero-energy excitations.

The domain wall induces non-zero one-point functions, which to the leading order in perturbation theory can be calculated by simply substituting the classical solution (2.6), (2.7) for the scalar fields $Y^{A}$ in (4.1). The outcome can be expressed as an overlap of the operator's wavefunction with a fixed state in the spin chain's Hilbert space, which we call MPS, the Matrix Product State:

$$
\langle\mathcal{O}(x)\rangle=\frac{1}{x^{L}} \frac{1}{\lambda^{L} L^{\frac{1}{2}}} \frac{\langle\mathrm{MPS} \mid \Psi\rangle}{\langle\Psi \mid \Psi\rangle^{\frac{1}{2}}} .
$$

The prefactor accounts for the difference between the spin-chain scalar product and the norm defined by the two-point correlation function. The Matrix Product State is built from the matrices (2.7) defining the classical solution:

$$
\operatorname{MPS}_{A_{2} \ldots A_{2 L}}^{A_{1} \ldots A_{2 L-1}}=\operatorname{tr} S^{A_{1}} S_{A_{2}}^{\dagger} \ldots S^{A_{2 L-1}} S_{A_{2 L}}^{\dagger} .
$$

The state so defined has non-zero components only when the indices $A_{l}$ take values 1 or 2 denoted collectively by greek letters as above.

Two convenient representations of the boundary state arise upon combining even and odd sites of the spin chain as in (2.8) or (2.16):

$$
\begin{aligned}
& \text { MPS }=\operatorname{tr}_{\text {aux }} M_{12} \ldots M_{2 L-1,2 L}, \\
& \text { MPS }=\operatorname{tr}_{\text {aux }} \widehat{M}_{23} \ldots \widehat{M}_{2 L, 1} .
\end{aligned}
$$

The building blocks are matrices in the auxiliary space tensored with a quantum state of two neighboring spins:

$$
\begin{aligned}
M & =\frac{q}{2} \mathbb{1} \otimes \mathbb{1}+t_{i} \otimes \sigma^{i}, \\
\widehat{M} & =\frac{q-1}{2} \mathbb{1} \otimes \mathbb{1}-\hat{t}^{i} \otimes \sigma_{i} .
\end{aligned}
$$

Here $t_{i}$ (or $\hat{t}^{i}$ ) and the first $\mathbb{1}$ act in the auxiliary space of dimension $q-1$ (or $q$ ), while $\sigma^{i}$ and the second $\mathbb{1}$ represent the state in the quantum space of two neighboring sites of the spin chain. 
When $q=2$, one of the two $\mathfrak{s u}(2)$ representations is trivial: $t_{i}=0$. There is no auxiliary space to trace over and the boundary state becomes two-site entangled:

$$
\mathrm{MPS}_{2} \underset{\alpha_{2} \ldots \alpha_{2 L}}{\alpha_{1} \ldots \alpha_{2 L-1}}=\delta_{\alpha_{2}}^{\alpha_{1}} \ldots \delta_{\alpha_{2 L}}^{\alpha_{2 L-1}}
$$

in other words it is a Valence Bond State:

$$
\left\langle\mathrm{MPS}_{2}\right|=\left\langle\left. K\right|^{\otimes L} \equiv\langle\mathrm{VBS}|,\right.
$$

where $K$ is a two-site state with components

$$
K_{\beta}^{\alpha}=\delta_{\beta}^{\alpha} .
$$

Degeneration of a matrix product state into a valence bond state is reminiscent to a similar phenomenon in the $\mathfrak{s u}(2)$ sector of $\mathcal{N}=4 \mathrm{SYM}[39]$.

\subsection{Selection rules}

We can now determine what type of single trace operators get non-vanishing one-point functions at tree-level. Obviously, the relevant operators must be built entirely from fields of the type $Y^{1}, Y^{2}, Y_{1}^{\dagger}, Y_{2}^{\dagger}$. Among these is the chiral primary

$$
\mathcal{O}=\operatorname{Tr}\left(Y^{1} Y_{2}^{\dagger} \ldots Y^{1} Y_{2}^{\dagger}\right)
$$

the vacuum of the spin chain. We find from (2.8), (2.10) and (2.13):

$$
Y^{1} Y_{2}^{\dagger}=\Phi_{2}^{1}=\frac{t^{i} \sigma_{i 2}^{1}}{x}=\frac{2 t^{-}}{x}
$$

meaning that $Y^{1} Y_{2}^{\dagger}$ is lower triangular and the traces of all of its powers vanish. This is in contrast to the parallel study in $\mathcal{N}=4 \mathrm{SYM}$ where the corresponding chiral primary (vacuum of the spin chain) has a non-vanishing one-point function [2]. This fact should be visible in a string theory analysis as well. For the other two-site combinations of fields we find

$$
\begin{aligned}
Y^{2} Y_{1}^{\dagger} & =\frac{2 t^{+}}{x} \\
Y^{1} Y_{1}^{\dagger} & =\frac{q+2 t^{3}}{2 x}, \\
Y^{2} Y_{2}^{\dagger} & =\frac{q-2 t^{3}}{2 x} .
\end{aligned}
$$

We thus see that operators with non-vanishing tree-level vevs can contain an arbitrary number of field combinations of the type $\left(Y^{1} Y_{1}^{\dagger}\right)$ and $\left(Y^{2} Y_{2}^{\dagger}\right)$ whereas a combination $\left(Y^{1} Y_{2}^{\dagger}\right)$ requires another term of the type $\left(Y^{2} Y_{1}^{\dagger}\right)$.

With the vacuum given by (4.12) we see that in order to get a non-vanishing one-point function we need to have a number of excitations which is equal to half the length of the spin chain. Among these, there is a particular symmetric set-up where we have the same number of excitations on the odd sites of the spin chain as on the even sites. Denoting 
the length of the alternating spin chain as $2 \mathrm{~L}$ and using the distinguished Dynkin diagram of the underlying super Lie algebra these states are characterized by having the number of Bethe roots (see below) on the three relevant nodes of the diagram identical and all equal to $L$ :

$$
K_{1}=K_{2}=K_{3}=L
$$

In order to evaluate the one-point functions of these operators we need to construct the appropriate eigenstates of the alternating spin chain. This problem was analyzed in [16], see also [18].

A state with $K_{i}$ Bethe roots belongs to the $\mathfrak{s u}(4)$ representation with the Dynkin labels

$$
\left[L-2 K_{1}+K_{2}, K_{1}-2 K_{2}+K_{3}, L-2 K_{3}+K_{2}\right]
$$

Consequently, (4.17) describes a singlet. This should not come as a surprise. The domain wall (equivalently, the boundary state in the spin chain) breaks $\mathrm{SU}(4)$ to $\mathrm{SU}(2) \times \mathrm{SU}(2) \times$ $\mathrm{U}(1)$. An $\mathrm{SU}(4)$ singlet is a singlet of this smaller group and is thus allowed to have a non-zero overlap with the boundary state. Singlets of $\mathrm{SU}(2) \times \mathrm{SU}(2) \times \mathrm{U}(1)$ do exist in non-trivial representations of SU(4) and they have non-zero one-point functions, but these states are not $\mathrm{SU}(4)$ primaries and if we concentrate on highest weights, as typically done in the Bethe ansatz, then (4.17) is a necessary condition for the one-point function not to vanish.

An example of non-singlet operator with a non-trivial one-point function is a chiral primary

$$
\mathcal{O}_{\mathbf{n}, \mathbf{m}}=\operatorname{tr}\left(n_{A}^{\dagger} Y^{A} m^{B} Y_{B}^{\dagger}\right)^{L}
$$

The complex vectors $\mathbf{n}$ and $\mathbf{m}$ must satisfy $\left(\mathbf{n}^{\dagger} \cdot \mathbf{m}\right)=0$. As follows from (4.5), (4.9), for $q=2$ the one-point function is

$$
\left\langle\mathcal{O}_{\mathbf{n}, \mathbf{m}}\right\rangle=\frac{1}{x^{L}} \frac{1}{\lambda^{L} L^{\frac{1}{2}}} \frac{\left(\widetilde{\mathbf{n}}^{\dagger} \cdot \widetilde{\mathbf{m}}\right)^{L}}{\left(\mathbf{n}^{\dagger} \cdot \mathbf{n}\right)^{\frac{L}{2}}\left(\mathbf{m}^{\dagger} \cdot \mathbf{m}\right)^{\frac{L}{2}}}
$$

where $\widetilde{\mathbf{n}}^{\dagger}=\left(n_{1}^{\dagger}, n_{2}^{\dagger}\right), \widetilde{\mathbf{m}}=\left(m_{1}, m_{2}\right)$, i.e. complex vectors with only two components. Obviously $\left(\mathbf{n}^{\dagger} \cdot \mathbf{m}\right)=0$ does not imply $\left(\widetilde{\mathbf{n}}^{\dagger} \cdot \widetilde{\mathbf{m}}\right)=0$. We now characterize $\mathrm{SU}(2) \times \mathrm{SU}(2) \times$ $\mathrm{U}(1)$-invariant chiral primaries and their one-point functions more precisely.

\subsection{Chiral primary operators at weak coupling}

The chiral primary operators are in one-to-one correspondence with the spherical harmonics on $\mathbb{C} P^{3}$ and only those which carry the $\mathrm{SU}(2) \times \mathrm{SU}(2) \times \mathrm{U}(1)$ symmetry of the classical fields will have non-vanishing one-point functions. As noticed earlier, there is only one chiral primary with this symmetry for each even value of the length. We can read off the relevant operators from the spherical harmonics that we determined in section 3.3. To do so we first rewrite these as homogeneous polynomials in $\cos \xi$ and $\sin \xi$. For the first few 
cases we get

$$
\begin{aligned}
& Y_{1}(\xi)=\mathcal{N}_{1}\left(-\cos ^{2} \xi+\sin ^{2} \xi\right), \\
& Y_{2}(\xi)=\mathcal{N}_{2}\left(\cos ^{4} \xi-3 \cos ^{2} \xi \sin ^{2} \xi+\sin ^{4} \xi\right), \\
& Y_{3}(\xi)=\mathcal{N}_{3}\left(-\cos ^{6} \xi+6 \cos ^{4} \xi \sin ^{2} \xi-6 \sin ^{4} \xi \cos ^{2} \xi+\sin ^{6} \xi\right), \\
& Y_{4}(\xi)=\mathcal{N}_{4}\left(\cos ^{8} \xi-10 \cos ^{6} \xi \sin ^{2} \xi+20 \cos ^{4} \xi \sin ^{4} \xi-10 \cos ^{2} \xi \sin ^{6} \xi+\sin ^{8} \xi\right) .
\end{aligned}
$$

Secondly, we make the replacements $\cos ^{2} \xi \rightarrow Y^{1} Y_{1}^{\dagger}+Y^{2} Y_{2}^{\dagger}$ and $\sin ^{2} \xi \rightarrow Y^{3} Y_{3}^{\dagger}+Y^{4} Y_{4}^{\dagger}$ and symmetrize the resulting powers of fields. For $Y_{1}(\xi)$ we trivially get

$$
Y_{1}(\xi)=\mathcal{N}_{1} \operatorname{Tr}\left(Y^{3} Y_{3}^{\dagger}+Y^{4} Y_{4}^{\dagger}-Y^{1} Y_{1}^{\dagger}-Y^{2} Y_{2}^{\dagger}\right)
$$

Moving on to $Y_{2}(\xi)$ we find

$$
Y_{2}(\xi)=\mathcal{N}_{2} C_{I_{1} I_{2}}^{J_{1} J_{2}} \operatorname{Tr} Y^{I_{1}} Y_{J_{1}}^{\dagger} Y^{I_{2}} Y_{J_{2}}^{\dagger},
$$

with

$$
\begin{array}{r}
C_{11}^{11}=C_{22}^{22}=C_{33}^{33}=C_{44}^{44}=1, \\
C_{12}^{12}=C_{12}^{21}=C_{21}^{12}=C_{21}^{21}=C_{34}^{34}=C_{34}^{43}=C_{43}^{34}=C_{43}^{43}=\frac{1}{2}, \\
C_{13}^{13}=C_{13}^{31}=C_{31}^{13}=C_{31}^{31}=C_{14}^{14}=C_{14}^{41}=C_{41}^{14}=C_{41}^{41}=-\frac{3}{4}, \\
C_{23}^{23}=C_{23}^{32}=C_{32}^{23}=C_{32}^{32}=C_{24}^{24}=C_{24}^{42}=C_{42}^{24}=C_{42}^{42}=-\frac{3}{4}
\end{array}
$$

and thus $C$ being a symmetric traceless tensor as required. Only the components with indices taking values 1 or 2 will be of importance for the one-point functions but all components play a role when it comes to normalization. Requiring the operators to be unit normalized, i.e.

$$
C_{I_{1} I_{2} \ldots I_{L}}^{J_{1} J_{2} \ldots J_{L}} C_{J_{1} J_{2} \ldots J_{L}}^{I_{1} I_{2} \ldots I_{L}}=1
$$

gives the following normalization constant

$$
\mathcal{N}_{L}=\left(\begin{array}{c}
2 L+2 \\
L
\end{array}\right)^{-1 / 2}
$$

and leads to the following normalization of the spherical harmonics on the string theory side

$$
\int_{S^{7} / \mathbb{Z}_{k}} Y_{L}\left(Y_{K}\right)^{*}=\delta_{L K} 2 \pi^{4} \frac{(L !)^{2}}{(2 L+3) !} .
$$

The one point function of these chiral primaries can be found by direct computation and reads for the first few cases (excluding the field theoretical prefactor that is reinstated 
below in eq. (4.33))

$$
\begin{aligned}
\left\langle Y_{1}\right\rangle & =\frac{1}{2}\left(q^{2}-q\right), \\
\left\langle Y_{2}\right\rangle & =\frac{1}{\sqrt{15}}\left(q^{3}-\frac{3}{2} q^{2}+\frac{1}{2} q\right), \\
\left\langle Y_{3}\right\rangle & =\frac{1}{\sqrt{56}}\left(q^{4}-2 q^{3}+q^{2}\right), \\
\left\langle Y_{4}\right\rangle & =\frac{1}{\sqrt{210}}\left(q^{5}-\frac{5}{2} q^{4}+\frac{5}{3} q^{3}-\frac{1}{6} q\right) \\
\left\langle Y_{5}\right\rangle & =\frac{1}{\sqrt{792}}\left(q^{6}-3 q^{5}+\frac{5}{2} q^{4}-\frac{1}{2} q^{2}\right), \\
\left\langle Y_{6}\right\rangle & =\frac{1}{\sqrt{3003}}\left(q^{7}-\frac{7}{2} q^{6}+\frac{7}{2} q^{5}-\frac{7}{6} q^{3}+\frac{1}{6} q\right),
\end{aligned}
$$

which is compatible with the following general expression.

$$
\left\langle Y_{L}\right\rangle=\frac{1}{x^{L}} \frac{1}{\lambda^{L} L^{1 / 2}} \mathcal{N}_{L}(L+1) \sum_{a=1}^{q-1} a^{L} .
$$

\section{$5 \quad$ Excited states}

We now turn to studying one-point functions of non-protected operators starting with the scalar sector in subsection 5.1 and moving on the full theory in subsection 5.2.

\section{$5.1 \quad \mathrm{SU}(4)$ sector}

The spectrum of the SU(4) spin chain (4.2) is described by the standard group-theoretic Bethe equations

$$
\begin{aligned}
\left(\frac{u_{a j}-\frac{i q_{a}}{2}}{u_{a j}+\frac{i q_{a}}{2}}\right)^{L} \prod_{b k} \frac{u_{a j}-u_{b k}+\frac{i M_{a b}}{2}}{u_{a j}-u_{b k}-\frac{i M_{a b}}{2}} & \equiv \mathrm{e}^{i \chi_{a j}}=(-1)^{\frac{M_{a a}}{2}}, \\
E & =\lambda^{2} \sum_{a j} \frac{q_{a}}{u_{a j}^{2}+\frac{q_{a}^{2}}{4}},
\end{aligned}
$$

specified to the SU(4) Cartan matrix and the Dynkin labels of the spin representation:

$$
M=\left[\begin{array}{ccc}
2 & -1 & 0 \\
-1 & 2 & -1 \\
0 & -1 & 2
\end{array}\right], \quad q=\left[\begin{array}{l}
1 \\
0 \\
1
\end{array}\right]
$$

The hallmark of boundary integrability is a $\mathbb{Z}_{2}$ symmetry acting on the Bethe roots

$$
\Omega: u_{a j} \rightarrow-u_{\sigma(a) j} .
$$

Only invariant Bethe states have a non-zero overlap with the boundary state at hand, (4.6) in our case. This can be regarded a defining property of a boundary state that makes it consistent with the underlying integrability structure $[39,40]$. 
The boundary state is called chiral if parity acts trivially on the Dynkin diagram ( $\sigma=\mathrm{id}$ ), and achiral otherwise [5, 41]. The permutation $\sigma$ should anyway square to the identity: $\sigma^{2}=$ id and be a symmetry of the Bethe equations: $M_{\sigma(a) \sigma(b)}=M_{a b}$ and $q_{\sigma(a)}=q_{a}$. The boundary state we consider is actually achiral, with parity interchanging the momentum-carrying nodes:

$$
\sigma=(13)
$$

The $\mathbb{Z}_{2}$ transformation represents the spacial reflection of the spin chain, or charge conjugation in terms of the original field-theory operators. The ABJM spin chain hosts complex representations $\mathbf{4}$ and $\overline{\mathbf{4}}$ at alternate sites and those get interchanged by parity. The reflection (5.5) is the complex conjugation acting on the Dynkin diagram and therefore must accompany inversion of rapidities.

Parity ordains a symmetrically paired structure of the Bethe roots: $\left\{u_{a j},-u_{\sigma(a) j}\right\}$. Solitary zero roots are also allowed on the nodes with $\sigma(a)=a$, being fixed points of parity $\Omega$. The key word here is solitary. Paired roots can accidentally take zero values, namely it can happen that $\left\{0_{a}, 0_{\sigma(a)}\right\}, \sigma(a) \neq a$ is part of the root configuration consistent with the Bethe equations. These accidental zeros are not fixed points of parity and do not require special treatment. When talking about zero roots in what follows we specifically mean unpaired roots on the nodes invariant under parity transformation. A parity-invariant state can thus be characterized by half of the paired roots $u_{a j}$ and the nodes $a_{\alpha}$ hosting solitary zero roots. ${ }^{1}$

Overlaps of integrable boundary states with Bethe eigenstates are described by a remarkably compact formula, which is universal to a large degree albeit its detailed structure is model-specific and is actually known on a case-by-case basis. A systematic derivation of the overlap formulas goes back to the work of Tsuchiya [42] and has so far been restricted to $\mathfrak{s u}(2)[43,44]$ (see also [3, 45]). Known overlaps for higher-rank groups are based on educated guesswork aided by symmetry consideration $[8,9,46]$, while a systematic derivation has only appeared recently for a class of $\mathrm{SU}(N)$ spin chains [11]. Fortunately, the $\mathrm{SU}(4)$ spin chain of ABJM is precisely of the type considered in [11]. In fact, the overlap for the simplest case of VBS (4.10), (4.11) is explicitly given in [11]: ${ }^{2}$

$$
\frac{\langle\mathrm{VBS} \mid \mathbf{u}\rangle}{\langle\mathbf{u} \mid \mathbf{u}\rangle^{\frac{1}{2}}}=2^{-L} Q_{2}(i) \sqrt{\frac{\operatorname{Sdet} G}{Q_{2}(0) Q_{2}\left(\frac{i}{2}\right)}} .
$$

\footnotetext{
${ }^{1}$ In the SU(4) case solitary zero roots can only sit on the middle node. We prefer to keep the discussion general in view of eventual generalization to the full superconformal group.

${ }^{2}$ It was conjectured in [11] that the overlap formula derived there should describe domain walls in ABJM theory. This conjecture turns out to be correct. In fact, the overlap formula in [11] applies to a larger class of valence-bond states that form a one-parameter family with the two-site matrix $\mathcal{K}(a)=1+a \sigma_{1} \otimes \sigma_{1}$ which for $a=1$ is related to (4.11) by an $\mathrm{SU}(4)$ transformation: $K=S^{\dagger} U^{\dagger} \mathcal{K}(1) U S$ with $U=\left(1-i \sigma_{2}\right) \otimes\left(1-i \sigma_{2}\right) / 2$ and $S$ representing the (24) permutation. It would be interesting to understand if the domain-wall solution admits a one-parameter deformation that corresponds to the boundary states with $a \neq 1$. The twosite matrix $\mathcal{K}(a)$ is non-degenerate for $a \neq \pm 1$ and the putative domain wall solution should involve all four scalars. The string dual is then expected to be a D8 brane wrapping the whole $\mathbb{C} P^{3}$. Candidate brane configurations have been considered in [47], and it would be interesting to find their field-theory counterparts.
} 
The key ingredient of the overlap formula is the superdeterminant of the Gaudin matrix

$$
G_{a j, b k}=\frac{\partial \chi_{a j}}{\partial u_{b k}}
$$

where the $\mathbb{Z}_{2}$ grading of the superdeterminant is defined by the parity transformation (5.4) acting on rows and columns of the Gaudin matrix. In the basis where parity acts diagonally the superdeterminant becomes a ratio of ordinary determinants:

$$
\operatorname{Sdet} G=\frac{\operatorname{det} G^{+}}{\operatorname{det} G^{-}}
$$

The rows and columns of $G^{-}$are labeled by positive Bethe roots only, while $G^{+}$includes in addition the zero roots:

$$
\begin{aligned}
G_{a j, b k}^{ \pm} & =\left(\frac{L q_{a}}{u_{a j}^{2}+\frac{q_{a}^{2}}{4}}-\sum_{c l} K_{a j, c l}^{+}-\frac{1}{2} \sum_{\alpha} K_{a j, a_{\alpha} 0}^{+}\right) \delta_{a b} \delta_{j k}+K_{a j, b k}^{ \pm} \\
G_{a j, \alpha}^{+} & =\frac{1}{\sqrt{2}} K_{a j, a_{\alpha} 0}^{+}, \\
G_{\alpha \beta}^{+} & =\left(\frac{4 L}{q_{q_{\alpha}}}-\sum_{c l} K_{a_{\alpha} 0, c l}^{+}-\sum_{\gamma} \frac{4}{M_{a_{\alpha} a_{\gamma}}}\right) \delta_{\alpha \beta}+\frac{4}{M_{a_{\alpha} a_{\beta}}}
\end{aligned}
$$

where

$$
K_{a j, b k}^{ \pm}=\frac{M_{a b}}{\left(u_{a j}-u_{b k}\right)^{2}+\frac{M_{a b}^{2}}{4}} \pm \frac{M_{a \sigma(b)}}{\left(u_{a j}+u_{b k}\right)^{2}+\frac{M_{a \sigma(b)}^{2}}{4}} .
$$

Potentially ill-defined quantities, like $1 / M_{a b}$ for $M_{a b}=0$, are declared to be zero.

The Gaudin factor in the overlap formula is decorated by the Baxter polynomials:

$$
Q_{a}(u)=\prod_{\text {all }} \prod_{\text {roots }}\left(u-u_{a j}\right) .
$$

To be more precise, the Q-functions in the overlap formula are regularized by omitting eventual zero roots. Hopefully the use of the same notations for the full and regularized Baxter polynomials will not cause any confusion.

Together with (4.5), the overlap formula (5.6) describes the tree-level expectation values of all scalar operators. ${ }^{3}$ Before extending the overlap formula to the full set of operators, we consider a few representative examples.

As discussed in section 4.2, primaries with non-zero overlaps must be $\mathrm{SU}(4)$ singlets. There are two singlets at length two, the simplest possible case. The Bethe-ansatz description of one of them is singular, involving roots at $\pm i / 2$ [16]. The Gaudin matrix then has to be regularized and redefined. The correct prescription of absorbing infinities is known [50], but its detailed outline would create unnecessary complications and since our goals are mostly illustrative, we concentrate on the other operator

$$
\mathcal{O}_{2}=\operatorname{tr}\left(Y^{A} Y_{A}^{\dagger} Y^{B} Y_{B}^{\dagger}+Y^{A} Y_{B}^{\dagger} Y^{B} Y_{A}^{\dagger}\right)
$$

\footnotetext{
${ }^{3}$ As it stands, the formula applies to conformal primaries. Descendants acquire an extra group-theoretic factor [48, 49], which we do not display here.
} 
having energy $10 \lambda^{2}$ and being described by regular Bethe roots [16]:

$$
u_{1}=\left\{\sqrt{\frac{3}{20}},-\sqrt{\frac{3}{20}}\right\}=u_{3}, \quad u_{2}=\left\{\frac{1}{\sqrt{5}},-\frac{1}{\sqrt{5}}\right\} .
$$

Taking the positive roots, one per node, to represent the state: ${ }^{4}$

$$
u_{a j}=\{\{u\},\{v\},\{u\}\},
$$

we get for the Gaudin factors defined in (5.9):

$$
G^{ \pm}=\left[\begin{array}{ccc}
\frac{3}{2} \frac{1}{\frac{1}{4}+u^{2}}+\kappa^{+} & -\kappa^{ \pm} & \pm \frac{1}{2} \frac{1}{\frac{1}{4}+u^{2}} \\
-\kappa^{ \pm} & 2 \kappa^{+}-\frac{1}{2} \frac{1 \mp 1}{\frac{1}{4}+v^{2}} & -\kappa^{ \pm} \\
\pm \frac{1}{2} \frac{1}{\frac{1}{4}+u^{2}} & -\kappa^{ \pm} & \frac{3}{2} \frac{1}{\frac{1}{4}+u^{2}}+\kappa^{+}
\end{array}\right]
$$

where

$$
\kappa^{ \pm}=\frac{1}{\frac{1}{4}+(u-v)^{2}} \pm \frac{1}{\frac{1}{4}+(u+v)^{2}} .
$$

Setting $u=\sqrt{3 / 20}, v=1 / \sqrt{5}$, and calculating the determinants we find:

$$
\operatorname{Sdet} G \equiv \frac{\operatorname{det} G^{+}}{\operatorname{det} G^{-}}=\frac{9}{10} .
$$

The one-point function follows from (5.6), (4.5):

$$
\left\langle\mathcal{O}_{2}(x)\right\rangle=\frac{1}{x^{2}} \frac{3}{2 \sqrt{5} \lambda^{2}} .
$$

Properly normalizing the operator and replacing the fields in (5.12) by the classical solution gives, of course, the same result.

Another example is a length-three operator

$$
\begin{aligned}
\mathcal{O}_{3}= & (\sqrt{10}-1) \operatorname{tr}\left(Y^{A} Y_{A}^{\dagger} Y^{B} Y_{B}^{\dagger} Y^{C} Y_{C}^{\dagger}+Y^{A} Y_{C}^{\dagger} Y^{B} Y_{A}^{\dagger} Y^{C} Y_{B}^{\dagger}\right. \\
& \left.-Y^{A} Y_{B}^{\dagger} Y^{B} Y_{C}^{\dagger} Y^{C} Y_{A}^{\dagger}\right)+9 \operatorname{tr} Y^{A} Y_{A}^{\dagger} Y^{B} Y_{C}^{\dagger} Y^{C} Y_{B}^{\dagger},
\end{aligned}
$$

with the anomalous dimension $(8+2 \sqrt{10}) \lambda^{2}$ and root content

$$
u_{1}=\{\alpha,-\alpha, 0\}=u_{3}, \quad u_{2}=\left\{\frac{2 \alpha}{\sqrt{3}},-\frac{2 \alpha}{\sqrt{3}}, 0\right\}, \quad \alpha^{2}=\sqrt{\frac{2}{5}}-\frac{1}{4} .
$$

It is easy to check that the operator is an eigenstate of the Hamiltonian (4.2) and that the root configuration solves the Bethe equations.

The zero roots on nodes 1 and 3 are accidental, they are paired with one another. On the contrary, the zero on node 2 is solitary and does not have a pair. Because of that the Gaudin factors have different sizes, $5 \times 5$ for $G^{+}$and $4 \times 4$ for $G^{-}$, see (5.9). We can represent pairs by $\{\{\alpha,-\alpha, 0\},\{2 \alpha / \sqrt{3}\},\{\}\}$, for example. Evaluating the determinants we find:

$$
\left\langle\mathcal{O}_{3}(x)\right\rangle=\frac{1}{x^{3}} \frac{1}{\lambda^{3}} \sqrt{\frac{54+17 \sqrt{10}}{390}} .
$$

We checked that replacement of scalar fields in the operator by the domain wall solution gives the same result.

\footnotetext{
${ }^{4}$ We can equally well represent pairs with $\{\{u,-u\},\{v\},\{\}\}$. This does not change the result.
} 


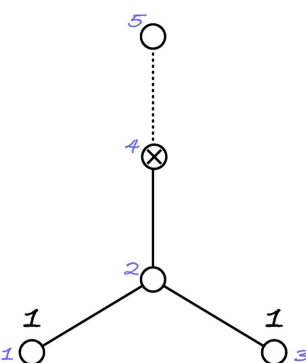

(a)

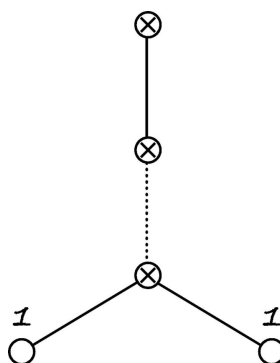

(b)

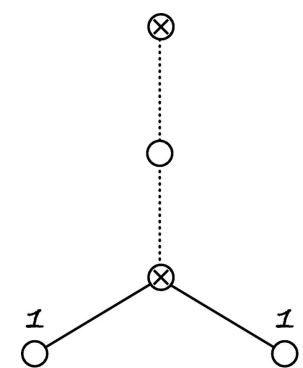

(c)

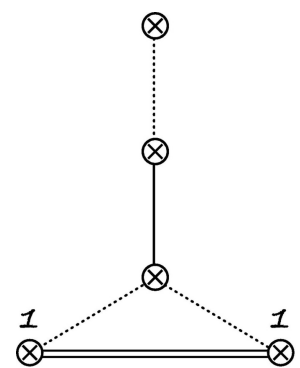

(d)

Figure 1. Dynkin diagram of $\mathfrak{o s p}(6 \mid 4)$ in different gradings.

\section{$5.2 \quad$ Full spectrum}

All possible operators in the ABJM theory are described by the $\operatorname{OSp}(6 \mid 4)$ Bethe equations. The Dynkin diagram is shown in figure 1(a), where as usual the matrix element for a solid link is $-1,+1$ for a broken link, 0 for a fermionic (crossed) node, +2 for a bosonic node between solid links and -2 between broken links. The spin representation has non-zero Dynkin indices on the nodes 1 and 3 as shown in the figure. Nodes 1, 2 and 3 correspond to the scalar $\mathrm{SU}(4)$ subsector discussed so far. An excitation on node 4 transforms a boson into a fermion and that on node 5 executes a spin flip.

\subsubsection{Fermionic dualities}

Since a Dynkin diagram for a superalgebra is not unique [51], the Bethe equations for a super-spin chain can be written in several different forms related by fermionic dualities [52]. For an example, consider the fermionic node in the original SU(4)-friendly diagram 1(a). The Bethe equations for the fermionic roots are

$$
1=\left.\frac{Q_{5}^{+} Q_{2}^{-}}{Q_{5}^{-} Q_{2}^{+}}\right|_{u_{4 j}}
$$

They can be equivalently represented as a QQ-relation between the Baxter polynomials:

$$
Q_{5}^{-} Q_{2}^{+}-Q_{5}^{+} Q_{2}^{-}=i\left(K_{2}-K_{5}\right) Q_{4} \widetilde{Q}_{4}
$$

where $Q_{4}$ is the original Q-function and $\widetilde{Q}_{4}$ is its complement that absorbes the remaining $K_{2}+K_{5}-K_{4}-1$ roots of the polynomial of the left-hand side. Here we use the standard notations for the argument shifts

$$
f^{[q]}(u)=f\left(u+\frac{i q}{2}\right), \quad f^{ \pm}=f^{[ \pm 1]}, \quad f^{ \pm \pm}=f^{[ \pm 2]} .
$$

While the Bethe equation (5.21) holds on the fermionic roots, the QQ-relation (5.22) is a functional equation valid for arbitrary value of the argument in $Q_{a}(u)$. It is easy to see that the roots of $\widetilde{Q}_{4}$, which we denote by $\widetilde{u}_{4 j}$, satisfy the same Bethe equations as $u_{4 j}$. 
Moreover, the scattering phases on the adjacent nodes can be expressed through $\widetilde{u}_{4 j}$ with the help of (5.22):

$$
\frac{Q_{4}^{+}}{Q_{4}^{-}}=-\left.\frac{Q_{2,5}^{++} \widetilde{Q}_{4}^{-}}{Q_{2,5}^{-}-\widetilde{Q}_{4}^{+}}\right|_{u_{2,5 j}} .
$$

The $Q_{2,5}^{ \pm \pm}$factors neatly cancel in the Bethe equations and we arrive at a new system associated with the Dynkin diagram in figure 1(b).

Dualizing further on node 5 we get the diagram in figure $1(\mathrm{c})$, in many respects distinguished. It is this diagram that allows for an extension to all loop orders [17].

The next step gives rise to a new feature. The QQ-relation on the central node contains a product of three Q-functions:

$$
Q_{4}^{-} Q_{1}^{+} Q_{3}^{+}-Q_{4}^{+} Q_{1}^{-} Q_{3}^{-}=i\left(K_{1}+K_{3}-K_{4}\right) Q_{2} \widetilde{Q}_{2} .
$$

As a consequence, the scattering phase in the Bethe equations for roots 1 and 3 contains an extra piece:

$$
\frac{Q_{2}^{+}}{Q_{2}^{-}}=-\left.\frac{Q_{1}^{++} Q_{3}^{++} \widetilde{Q}_{2}^{-}}{Q_{1}^{--} Q_{3}^{--} \widetilde{Q}_{2}^{+}}\right|_{u_{1,3 j}} .
$$

Substituting this into the Bethe equations for roots 1 and 3 not only cancels their selfscattering, but induces a mutual interaction depicted as a double line in figure 1(d). The Dynkin diagram ceases to be simply-laced.

\subsubsection{OSp $(6 \mid 4)$ overlaps}

The Gaudin superdeterminant transforms under fermionic dualities in a regular way, such that determinants constructed on the original and dual roots are related by a simple Jacobian. Namely, for the duality transformation on the $a$-th node, the following relation holds: ${ }^{5}$

$$
\prod_{b: M_{a b} \neq 0} Q_{b}(i / 2) \operatorname{Sdet} \widetilde{G}=Q_{a}(0) \widetilde{Q}_{a}(0) \operatorname{Sdet} G,
$$

where the product on the left-hand side is over all nodes adjacent to $a$. This formula was conjectured for $A_{n}$-type Dynkin diagrams and checked for a number of chiral boundary states $[15,50]$. The boundary state at hand is achiral and the Dynkin diagram is of the $D$-type, but we found that the formula continues to hold without change. Although we have no mathematical proof, we performed thorough numerical checks for all duality transformations considered in the previous subsection.

The transformation law of the Gaudin superdeterminant can be used to transform the overlap formula from one duality frame to another. The overlap of an integrable boundary state is expected to have a universal form

$$
\sqrt{\prod_{a} \frac{\prod_{j} Q_{a}\left(i \alpha_{a j} / 2\right)}{\prod_{k} Q_{a}\left(i \beta_{a k} / 2\right)} \operatorname{Sdet} G},
$$

\footnotetext{
${ }^{5}$ The formula seems to apply to simply-laced Dynkin diagrams, and may need to be modified in the non-simply-laced case, for example when dualizing on one of the nodes in figure $1(\mathrm{~d})$ connected by the double line.
} 


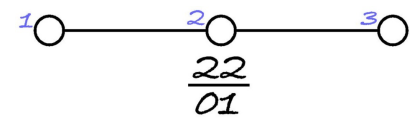

Figure 2. The SU(4) overlap formula in the graphic notations.
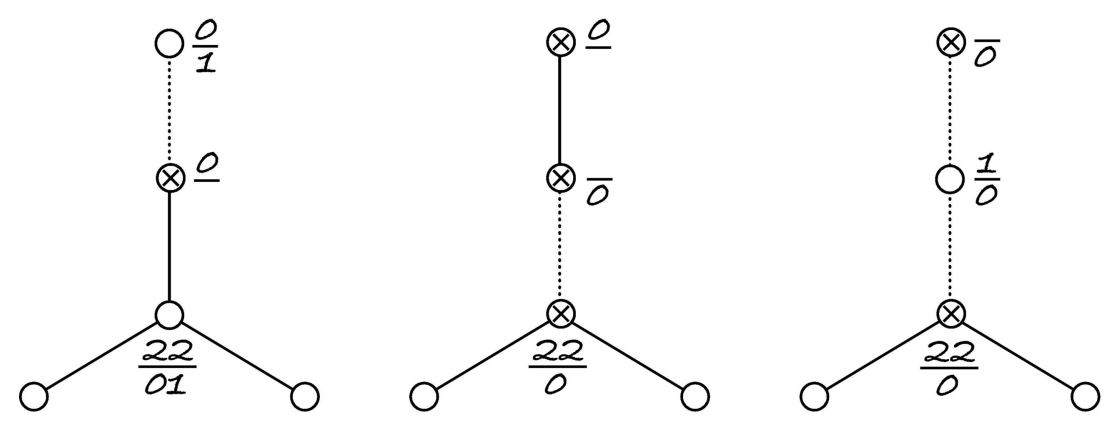

Figure 3. Overlap formulas in different grading.

or to be a linear combination of such terms. Ultimately, this structure is dictated by the underlying Algebraic Bethe Ansatz of rational type [3, 11]. But we simply accept this formula as an assumption. By itself this is not very constraining, but in conjunction with fermionic duality this structural assumption places powerful constraints on the particular values of the $\mathrm{Q}$-functions that can appear.

We use a graphic notation introduced in [15] for the basic building block (5.28) by placing numbers $\alpha_{a j}, \beta_{a k}$ directly on the Dynkin diagram:

$$
\frac{a_{a 1}^{a} \cdots a_{a n}}{\beta_{a 1} \cdots \beta_{a m}}
$$

For example, the overlap formula (5.6) for the $\mathrm{SU}(4)$ sectors is represented by figure 2 .

It is not hard to see that an extension to the full $\operatorname{OSp}(6 \mid 4)$ is actually unique as long as it respects fermionic duality. The transformation law (5.27) leaves two possibilities for a fermionic node, $0 /$ or $/ 0$, if the overlap is to retain its structure in the new frame. Otherwise the dependence on the original fermionic roots, that are traded for their duals, would not completely cancel. For the particular case at hand 0 / is preferable, the Jacobian then cancels $/ 1$ on the 2 nd node, and that is going to help in the subsequent steps. Since the 5 th node becomes fermionic as well, the only choice there is $0 / 1$. The $/ 1$ is neatly cancelled by the Jacobian leaving $0 /$ which can again be dualized.

Arguments of this kind reconstruct overlaps for the first three diagrams in figure 1 with the result shown in figure 3. Duality on the 4 th node of the first diagram replaces $Q_{4}(0) \operatorname{Sdet} G$ by $Q_{2}(i / 2) Q_{5}(i / 2) \operatorname{Sdet} \widetilde{G} / \widetilde{Q}_{4}(0)$ which gives the second diagram. Likewise, dualizing the 5 th node of the second diagram replaces $Q_{5}(0) \operatorname{Sdet} G$ by $Q_{4}(i / 2) \operatorname{Sdet} \widetilde{G} / \widetilde{Q}_{5}(0)$ yielding the third diagram. 

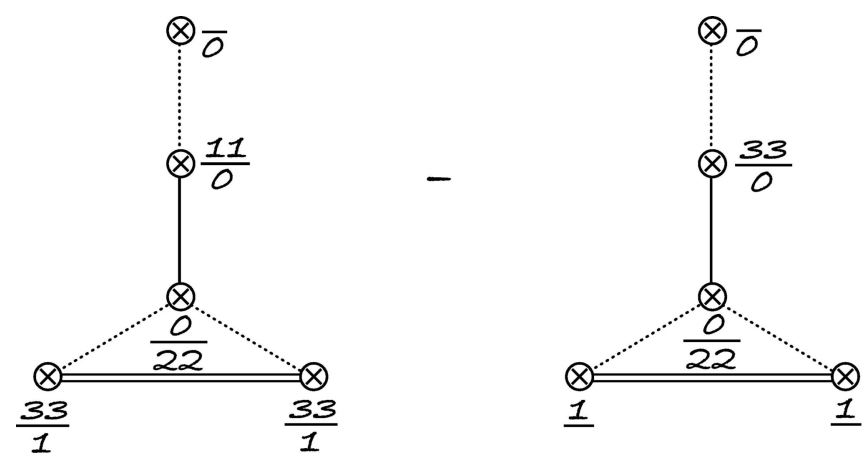

Figure 4. Overlap formula for a non-simply-laced form of the Dynkin diagram.

This diagram is of particular interest, because its Bethe equations can be extended to all orders in the coupling. The explicit form of the overlap for this distinguished grading is

$$
\frac{\left\langle\mathrm{MPS}_{2} \mid \mathbf{u}\right\rangle}{\langle\mathbf{u} \mid \mathbf{u}\rangle^{\frac{1}{2}}}=Q_{2}(i) \sqrt{\frac{Q_{4}(i / 2)}{Q_{2}(0) Q_{4}(0) Q_{5}(0)} \operatorname{Sdet} G} .
$$

We expect this formula to have an all-loop generalization, but we will not attempt to derive or guess it here.

It is also possible to dualize on the central node, albeit the outcome is somewhat different compared to the simply-laced case. The factor of $Q_{2}(i)$ is not accounted for by the Jacobian and has to be expressed through the other Q-functions, ${ }^{6}$ which is possible in virtue of the QQ-relation (5.25):

$$
\widetilde{Q}_{2}(i) \propto \frac{Q_{4}(i / 2) Q_{1}(3 i / 2) Q_{3}(3 i / 2)}{Q_{2}(i)}-\frac{Q_{4}(3 i / 2) Q_{1}(i / 2) Q_{3}(i / 2)}{Q_{2}(i)} .
$$

Standing out of the square root this factor does not compromise the general structure. The dualized overlap becomes a difference of two terms each of the canonical form, as illustrated in figure 4.

\section{One-point functions at strong coupling}

One-point functions at strong coupling can be calculated by a variant of the GKPW prescription $[53,54]$ which entails a fluctuation analysis of the supergravity background. The foundation for the fluctuation analysis for type IIA supergravity on $A d S_{4} \times S^{7}$ was laid in [55-57] and employed in the study the study three-point functions involving two giant gravitons and one tiny graviton in ABJM theory in $[58,59]$ as well as in the computation of correlation functions of vortex loop operators with local operators in [60].

Hence, let us consider the variation of the Euclidean DBI and WZ action. For the latter we have

$$
\delta I_{\mathrm{WZ}}=T_{4} \int d^{5} \sigma \mathcal{F} \wedge \mathcal{P}\left[\delta C_{3}\right]
$$

\footnotetext{
${ }^{6}$ The duality transformation must act from the diagram in 1 (d) to $1(\mathrm{c})$, so as to replace $Q_{2}(0)$ by $1 / \widetilde{Q}_{2}(0)$, and we need to express the dual Q-function through the original ones.
} 
where

$$
\left(\delta C_{3}\right)_{\mu_{1} \mu_{2} \mu_{3}}=\frac{R^{3}}{8} 2 \epsilon_{\mu_{1} \mu_{2} \mu_{3} \mu_{4}} \nabla^{\mu_{4}} s^{\Delta I}(X) Y_{\Delta I}(\Omega),
$$

with the $\mu$ 's referring to coordinates on $A d S_{4}$ and $\epsilon_{1234}=\sqrt{g_{A d S_{4}}}=z^{2}$. The $X$ likewise refers to $A d S_{4}$ coordinates while $\Omega$ refers to coordinates on $\mathbb{C} P^{3}$. For the relevant pull-back to the brane world-volume we get

$$
\begin{aligned}
\mathcal{P}\left[\delta C_{3}\right]_{01 z} & =\frac{R^{3}}{8} 2\left(x_{2}^{\prime}(z) \epsilon_{012 z} z^{2} \partial_{z}+\epsilon_{01 z 2} \frac{1}{z^{2}} \partial_{x_{2}}\right) s^{\Delta I}(X) Y_{\Delta I}(\Omega) \\
& =\frac{R^{3}}{8} 2 z^{2}\left(-Q \partial_{z}-\frac{1}{z^{2}} \partial_{x_{2}}\right) s^{\Delta I}(X) Y_{\Delta I}(\Omega) .
\end{aligned}
$$

We can now combine this with $\mathcal{F}$ and get

$$
\begin{aligned}
\delta I_{\mathrm{WZ}}= & T_{4} \widetilde{R}^{4} \sqrt{\alpha^{\prime}} k Q \int_{0}^{\infty} d z z^{2} \int_{-\infty}^{\infty} d x_{0} d x_{1} \int_{0}^{\pi} d \theta_{1} \sin \theta_{1} \int_{0}^{\pi} d \phi_{1} \\
& \times\left(-Q \partial_{z}-\frac{1}{z^{2}} \partial_{x_{2}}\right) s^{\Delta I}(X) Y_{\Delta I}(\Omega) .
\end{aligned}
$$

Moving on to the DBI action we find for the variation

$$
\delta I_{\mathrm{DBI}}=-T_{4} e^{-\Phi} \frac{1}{2} \int d^{5} \sigma \sqrt{\operatorname{det}(G+\mathcal{F})}(G+\mathcal{F})^{a b} \delta G_{a b},
$$

where for the AdS part of the metric we have

$$
\delta g_{\mu \nu}=\frac{4}{\Delta+2}\left[\nabla_{\mu} \nabla_{\nu}-\frac{1}{6} \Delta(\Delta-1) g_{\mu \nu}\right] s^{\Delta I}(X) Y_{\Delta I}(\Omega)
$$

whereas on $\mathbb{C} P^{3}$

$$
\delta g_{\alpha \beta}=\frac{\Delta}{3} g_{\alpha \beta} s^{\Delta I}(X) Y_{\Delta I}(\Omega) .
$$

To compute the double covariant derivative we will need some Christoffel symbols for $A d S_{4}$. The relevant ones are

$$
\Gamma_{00}^{z}=\Gamma_{11}^{z}=\Gamma_{22}^{z}=-z^{3}, \quad \Gamma_{z z}^{z}=-\frac{1}{z}, \quad \Gamma_{z 2}^{z}=\Gamma_{2 z}^{z}=\frac{1}{z},
$$

For the calculation of the variation we also need the inverse of the induced metric. Its non-zero relevant elements are

$$
\begin{aligned}
(G+\mathcal{F})^{00} & =(G+\mathcal{F})^{11}=\frac{1}{z^{2}}, & (G+\mathcal{F})^{z z} & =\left(\frac{1}{z^{2}}+z^{2}\left(x_{2}^{\prime}(z)\right)^{2}\right)^{-1}=\frac{z^{2}}{1+Q^{2}}, \\
(G+\mathcal{F})^{\theta_{1} \theta_{1}} & =\frac{1}{1+Q^{2}}, & (G+\mathcal{F})^{\phi_{1} \phi_{1}} & =\frac{1}{\sin ^{2} \theta_{1}\left(1+Q^{2}\right)},
\end{aligned}
$$

where we have set $\cos ^{2} \xi=1$. Of the fluctuations, only $\delta G_{z z}$ is a bit complicated involving a non-trivial pull back. From eq. (3.15) we get

$$
\sqrt{\operatorname{det}(G+\mathcal{F})}=\widetilde{R}^{5} z \sin \theta_{1}\left(1+Q^{2}\right),
$$


Putting everything together, we find

$$
\begin{aligned}
\delta I_{\mathrm{DBI}}= & -T_{4} \widetilde{R}^{4} k \sqrt{\alpha^{\prime}}\left(1+Q^{2}\right) \int_{0}^{\infty} d z z \int_{-\infty}^{\infty} d x_{0} d x_{1} \int_{0}^{2 \pi} d \phi_{1} \int_{0}^{\pi} d \theta_{1} \sin \theta_{1} \\
& \times\left\{\frac { 1 } { \Delta + 2 } \left[\frac{1}{z^{2}}\left(\partial_{0}^{2}+\partial_{1}^{2}\right)+3 z \partial_{z}\right.\right. \\
& \left.+\frac{z^{2}}{1+Q^{2}}\left(\partial_{z}^{2}-2 \frac{Q}{z^{2}}\left(\partial_{z} \partial_{2}-\frac{1}{z} \partial_{2}\right)+\frac{Q^{2}}{z^{4}} \partial_{2}^{2}\right)-\frac{1}{2} \Delta(\Delta-1)\right] \\
& \left.+\frac{\Delta}{6} \frac{1}{1+Q^{2}}\right\} s^{\Delta I}(X) Y_{\Delta I}(\Omega) .
\end{aligned}
$$

We note that the term in the last line originates from the spherical part of the geometry and the remaining terms come from the AdS part.

Implementing the GKPW prescription, i.e. differentiating after a delta-function source on the boundary coupling to a specific chiral primary CFT operator and setting the source to zero corresponds to picking out the term involving the corresponding spherical harmonic and replacing the mode $s_{\Delta I}(X)$ by its bulk to boundary propagator, i.e.

$$
\left\langle\mathcal{O}(x)_{\Delta}\right\rangle=-\delta I_{\mathrm{DBI}+\mathrm{WZ}} \mid s \rightarrow \frac{C_{\Delta}}{z^{\Delta}\left(\rho^{2}+\left(x_{2}-x\right)^{2}+1 / z^{2}\right)^{\Delta}}, \quad \rho^{2}=x_{0}^{2}+x_{1}^{2},
$$

where (see f.inst. [60])

$$
C_{\Delta}=\sqrt{k}\left(\frac{l_{p}}{R}\right)^{9 / 2} \mathcal{C}_{\Delta}
$$

with

$$
\mathcal{C}_{\Delta}=2^{\Delta / 2-1} \pi \frac{\Delta+2}{\Delta} \sqrt{\Delta+1} .
$$

Next step is to perform all the integrations and differentiations implied by the relations (6.6) and (6.12). Here one can check that the term with $\partial_{0}^{2}+\partial_{1}^{2}$ does not give any contribution and we can rewrite $\int_{-\infty}^{\infty} d x_{0} d x_{1}=2 \pi \int_{0}^{\infty} \rho d \rho$.

Furthermore, we notice that the common pre-factor of the two integrals can be rewritten as

$$
T_{4} \widetilde{R}^{4} k \sqrt{\alpha^{\prime}}=\frac{N}{8 \pi^{2}} .
$$

From (6.6) and (6.12) we immediately see that the standard chiral primary $\left(Y_{1} Y_{2}^{*}\right)^{L}$ has a vanishing one-point function as its evaluation involves the integral $\int_{0}^{2 \pi} d \phi_{1} \exp \left(i \phi_{1}\right)=0$. On the other hand the chiral primaries defined by the relation (3.25) have non-vanishing one-point functions.

Combining the factors (6.13) and (6.15) we see that in the strong coupling limit all one-point functions will carry a pre-factor

$$
\sim\left(\frac{l_{p}}{R}\right)^{9 / 2} \sqrt{k} N \sim k^{-3 / 2} \lambda^{-3 / 4} \sqrt{k} N \sim \lambda^{1 / 4}
$$

and since the integrals when expanded for large $Q$ involve only integer powers of $Q$ we will not immediately be able to compare to a perturbative gauge theory calculation even 
in the double scaling limit (3.24). This is in contrast to the $A d S_{5} \times S^{5}$ case where the strong coupling expansion in combination with a double scaling limit gave rise to one point functions expandable in integer powers of $\lambda[33,34]$.

Let us now calculate the one-point functions of the chiral primaries with symmetry $\mathrm{SU}(2) \times \mathrm{SU}(2) \times \mathrm{U}(1)$ given in eq. (3.28), see also (4.21). For these chiral primaries we can trivially perform the integration over $\phi_{1}$ and $\theta_{1}$ which gives a factor of $4 \pi$. Furthermore, our brane embedding is characterized by $\cos \xi=1$ so we get $Y_{\Delta}(\xi)=(-1)^{\Delta} \mathcal{N}_{\Delta}$ where we will ignore the overall phase. Summarizing, the one-point functions of our chiral primaries are given by

$$
\begin{aligned}
\left\langle Y_{\Delta}(x)\right\rangle= & \lambda^{1 / 4} \mathcal{N}_{\Delta} \mathcal{C}_{\Delta} \int_{0}^{\infty} d z z \int_{0}^{\infty} d \rho \rho\left\{\frac { 1 } { \Delta + 2 } \left[\left(3+Q^{2}(\Delta+5)\right) z \partial_{z}\right.\right. \\
& +z^{2}\left(\partial_{z}^{2}-2 \frac{Q}{z^{2}} \partial_{z} \partial_{2}-\frac{3}{z^{3}} Q \partial_{2}+\frac{Q^{2}}{z^{4}} \partial_{2}^{2}\right) \\
& \left.\left.-\frac{1}{2} \Delta(\Delta-1)\left(1+Q^{2}\right)\right]+\frac{\Delta}{6}\right\} \frac{1}{z^{\Delta}\left(\rho^{2}+\left(x_{2}-x\right)^{2}+1 / z^{2}\right)^{\Delta}} .
\end{aligned}
$$

We can now also integrate over $\rho$. This gives

$$
\begin{aligned}
\left\langle Y_{\Delta}(x)\right\rangle= & \frac{\lambda^{1 / 4} \mathcal{N}_{\Delta} \mathcal{C}_{\Delta}}{2(\Delta-1)} \int_{0}^{\infty} d z z\left\{\frac { 1 } { \Delta + 2 } \left[\left(3+Q^{2}(\Delta+5)\right) z \partial_{z}\right.\right. \\
& +z^{2}\left(\partial_{z}^{2}-2 \frac{Q}{z^{2}} \partial_{z} \partial_{2}+\frac{3}{z^{3}} Q \partial_{2}+\frac{Q^{2}}{z^{4}} \partial_{2}^{2}\right) \\
& \left.\left.-\frac{1}{2} \Delta(\Delta-1)\left(1+Q^{2}\right)\right]+\frac{\Delta}{6}\right\} \frac{1}{z^{\Delta}\left(\left(x_{2}-x\right)^{2}+1 / z^{2}\right)^{\Delta-1}},
\end{aligned}
$$

provided $\Delta>1$. The remaining integral is not convergent for $\Delta=2$. The same problem was observed for the $A d S_{5} \times S^{5}$ set-up [33, 34]. For $\Delta>2$, we find in the large-Q limit

$$
\left\langle Y_{\Delta}(x)\right\rangle \sim \lambda^{1 / 4} \frac{Q^{\Delta+1}}{x^{\Delta}} \sim \lambda^{-\Delta / 2-1 / 4} \frac{q^{\Delta+1}}{x^{\Delta}} .
$$

We notice that $x$ dependence is as expected for a defect one-point function and the leading power of $q$ is the same as on the gauge theory side. The powers of $\lambda$ on the two sides only agree in a large charge limit.

\section{Conclusion and outlook}

The D2-D4 domain wall version of ABJM theory shares many features with its D3-D5 counterpart in $\mathcal{N}=4$ super Yang Mills theory, most notably it provides us with novel examples of integrable boundary states within AdS/CFT and associated novel examples of exact overlap formulas. These integrable boundary states generically take the form of matrix product states with the bond dimension encoding the jump in the rank of the gauge group across the domain wall and degenerate to valence bond states as when the 
bond dimension becomes equal to one. Whereas the overlaps in the scalar sector of ABJM theory between Bethe eigenstates and matrix product states of bond dimension two could be read off from a recent result on integrable boundary states in alternating $\mathrm{SU}(N)$ spin chains [11], the relation to the valence bond states made it possible to extend the formula to the full theory by requiring covariance under fermionic duality transformations, thereby realizing the idea put forward in [50].

An obvious open problem is the extension of the overlap formula for matrix product states to general values of the bond dimension. For the integrable boundary states of relevance for $\mathcal{N}=4 \mathrm{SYM}$ this could be done using representation theory of twisted Yangians [12]. It would likewise be interesting to extend the overlap formulas to higher perturbative orders and eventually to the non-perturbative situation as well. One could envision a bootstrap based strategy in line with $[5,6]$ possibly with input from a direct perturbative calculation. The strategy for setting up the program for perturbative calculations would follow that employed for domain wall versions of $\mathcal{N}=4 \mathrm{SYM}$ where fuzzy spherical harmonics were used to disentangle the complicated mixing between color and flavor components of the scalar fields introduced by the non-trivial vacuum expectations values [36-38].

Considering the domain wall set-up in more detail from the string theory side calls for the development of improved methods. The strategy we used for the computation of overlaps works only for chiral primaries. As for the $A d S_{5} / C F T_{4}$ system a double scaling parameter naturally appears, but in the present case it does not allow for a precise comparison of the gauge and string theory results. One could hope that a version of supersymmetric localization makes it possible to compute overlaps exactly for particular chiral primaries as it was the case in $\mathcal{N}=4 \mathrm{SYM}[7,61,62]$. Interestingly, the operator algebra in the presence of the defect carries a natural integrability structure [63]. Furthermore, one could imagine an integrability based bootstrap procedure for overlaps formulated directly in the strong coupling language [7]. The D2-D4 probe brane set-up without flux does belongs to the list of integrable boundary conditions of [20] but it would be important to check that the model, like its D3-D5 probe brane cousin, remains integrable when the flux is introduced [64].

Ultimately, it would be interesting to arrive at a fully non-perturbative treatment of both the D2-D4 and the D3-D5 domain wall systems with the overlap formulas being given in terms of appropriate $g$-functions $[65,66]$.

Finally, it is possible that AdS/CFT contains even more integrable boundary states awaiting discovery.

\section{Acknowledgments}

We thank Matthias Volk for comments on the draft. The figures were prepared using the JaxoDraw package $[67,68]$. The work of CK was supported by DFF-FNU through grant number DFF-4002-00037. The work of KZ was supported by the grant "Exact Results in Gauge and String Theories" from the Knut and Alice Wallenberg foundation and by RFBR grant 18-01-00460A. 
Open Access. This article is distributed under the terms of the Creative Commons Attribution License (CC-BY 4.0), which permits any use, distribution and reproduction in any medium, provided the original author(s) and source are credited.

\section{References}

[1] N. Beisert et al., Review of AdS/CFT Integrability: An Overview, Lett. Math. Phys. 99 (2012) 3 [arXiv: 1012.3982] [INSPIRE].

[2] M. de Leeuw, C. Kristjansen and K. Zarembo, One-point Functions in Defect CFT and Integrability, JHEP 08 (2015) 098 [arXiv:1506.06958] [INSPIRE].

[3] I. Buhl-Mortensen, M. de Leeuw, C. Kristjansen and K. Zarembo, One-point Functions in AdS/dCFT from Matrix Product States, JHEP 02 (2016) 052 [arXiv: 1512.02532] [INSPIRE].

[4] C. Kristjansen, D. Müller and K. Zarembo, Integrable boundary states in D3-D5 dCFT: beyond scalars, JHEP 08 (2020) 103 [arXiv: 2005.01392] [inSPIRE].

[5] T. Gombor and Z. Bajnok, Boundary states, overlaps, nesting and bootstrapping AdS/dCFT, JHEP 10 (2020) 123 [arXiv: 2004.11329] [INSPIRE].

[6] T. Gombor and Z. Bajnok, Boundary state bootstrap and asymptotic overlaps in AdS/dCFT, JHEP 03 (2021) 222 [arXiv:2006.16151] [INSPIRE].

[7] S. Komatsu and Y. Wang, Non-perturbative defect one-point functions in planar $\mathcal{N}=4$ super-Yang-Mills, Nucl. Phys. B 958 (2020) 115120 [arXiv: 2004.09514] [INSPIRE].

[8] M. de Leeuw, C. Kristjansen and S. Mori, AdS/dCFT one-point functions of the SU(3) sector, Phys. Lett. B 763 (2016) 197 [arXiv:1607.03123] [INSPIRE].

[9] M. De Leeuw, C. Kristjansen and G. Linardopoulos, Scalar one-point functions and matrix product states of AdS/dCFT, Phys. Lett. B 781 (2018) 238 [arXiv:1802.01598] [INSPIRE].

[10] M. de Leeuw, C. Kristjansen and S. Mori, AdS/dCFT one-point functions of the SU(3) sector, Phys. Lett. B 763 (2016) 197 [arXiv:1607.03123] [INSPIRE].

[11] T. Gombor, On exact overlaps for $\mathfrak{g l}(N)$ symmetric spin chains, arXiv:2110.07960 [INSPIRE].

[12] M. De Leeuw, T. Gombor, C. Kristjansen, G. Linardopoulos and B. Pozsgay, Spin Chain Overlaps and the Twisted Yangian, JHEP 01 (2020) 176 [arXiv:1912.09338] [INSPIRE].

[13] N. Gromov, V. Kazakov, S. Leurent and D. Volin, Quantum spectral curve for arbitrary state/operator in $A d S_{5} / \mathrm{CFT}_{4}, J H E P 09$ (2015) 187 [arXiv:1405.4857] [INSPIRE].

[14] Z. Tsuboi, Solutions of the T-system and Baxter equations for supersymmetric spin chains, Nucl. Phys. B 826 (2010) 399 [arXiv:0906. 2039] [INSPIRE].

[15] C. Kristjansen, D. Müller and K. Zarembo, Overlaps and fermionic dualities for integrable super spin chains, JHEP 03 (2021) 100 [arXiv:2011.12192] [INSPIRE].

[16] J.A. Minahan and K. Zarembo, The Bethe ansatz for superconformal Chern-Simons, JHEP 09 (2008) 040 [arXiv:0806.3951] [InSPIRE].

[17] N. Gromov and P. Vieira, The all loop AdS4/CFT3 Bethe ansatz, JHEP 01 (2009) 016 [arXiv: 0807.0777] [INSPIRE].

[18] P. Yang, Y. Jiang, S. Komatsu and J.-B. Wu, Three-point functions in ABJM and Bethe Ansatz, JHEP 01 (2022) 002 [arXiv:2103.15840] [INSPIRE]. 
[19] Y. Jiang, S. Komatsu and E. Vescovi, Exact Three-Point Functions of Determinant Operators in Planar $N=4$ Supersymmetric Yang-Mills Theory, Phys. Rev. Lett. 123 (2019) 191601 [arXiv: 1907.11242] [INSPIRE].

[20] A. Dekel and Y. Oz, Integrability of Green-Schwarz Sigma Models with Boundaries, JHEP 08 (2011) 004 [arXiv: 1106.3446] [INSPIRE].

[21] O. Aharony, O. Bergman, D.L. Jafferis and J. Maldacena, $N=6$ superconformal Chern-Simons-matter theories, M2-branes and their gravity duals, JHEP 10 (2008) 091 [arXiv: 0806.1218] [INSPIRE].

[22] M. Benna, I. Klebanov, T. Klose and M. Smedback, Superconformal Chern-Simons Theories and $A d S_{4} / C F T_{3}$ Correspondence, JHEP 09 (2008) 072 [arXiv:0806.1519] [INSPIRE].

[23] S. Terashima, On M5-branes in N=6 Membrane Action, JHEP 08 (2008) 080 [arXiv: 0807.0197] [INSPIRE].

[24] A. Basu and J.A. Harvey, The M2-M5 brane system and a generalized Nahm's equation, Nucl. Phys. B 713 (2005) 136 [hep-th/0412310] [INSPIRE].

[25] W. Nahm, A Simple Formalism for the BPS Monopole, Phys. Lett. B 90 (1980) 413 [INSPIRE].

[26] D.-E. Diaconescu, D-branes, monopoles and Nahm equations, Nucl. Phys. B 503 (1997) 220 [hep-th/9608163] [INSPIRE].

[27] N.R. Constable, R.C. Myers and O. Tafjord, The Noncommutative bion core, Phys. Rev. D 61 (2000) 106009 [hep-th/9911136] [INSPIRE].

[28] D. Gaiotto and E. Witten, S-duality of Boundary Conditions In $N=4$ Super Yang-Mills Theory, Adv. Theor. Math. Phys. 13 (2009) 721 [arXiv:0807.3720] [InSPIRE].

[29] H. Nastase, C. Papageorgakis and S. Ramgoolam, The Fuzzy $S^{2}$ structure of M2-M5 systems in ABJM membrane theories, JHEP 05 (2009) 123 [arXiv:0903.3966] [INSPIRE].

[30] H. Nastase and C. Papageorgakis, Fuzzy Killing Spinors and Supersymmetric D4 action on the Fuzzy 2-sphere from the ABJM Model, JHEP 12 (2009) 049 [arXiv:0908.3263] [INSPIRE].

[31] B. Chandrasekhar and B. Panda, Brane Embeddings in $A d S_{4} \times C P^{3}$, Int. J. Mod. Phys. A 26 (2011) 2377 [arXiv:0909.3061] [INSPIRE].

[32] T. Nishioka and T. Takayanagi, On Type IIA Penrose Limit and $N=6$ Chern-Simons Theories, JHEP 08 (2008) 001 [arXiv:0806.3391] [INSPIRE].

[33] K. Nagasaki and S. Yamaguchi, Expectation values of chiral primary operators in holographic interface CFT, Phys. Rev. D 86 (2012) 086004 [arXiv:1205.1674] [InSPIRE].

[34] C. Kristjansen, G.W. Semenoff and D. Young, Chiral primary one-point functions in the D3-D7 defect conformal field theory, JHEP 01 (2013) 117 [arXiv:1210.7015] [INSPIRE].

[35] I. Buhl-Mortensen, M. de Leeuw, A.C. Ipsen, C. Kristjansen and M. Wilhelm, One-loop one-point functions in gauge-gravity dualities with defects, Phys. Rev. Lett. 117 (2016) 231603 [arXiv: 1606.01886] [INSPIRE].

[36] I. Buhl-Mortensen, M. de Leeuw, A.C. Ipsen, C. Kristjansen and M. Wilhelm, A Quantum Check of AdS/dCFT, JHEP 01 (2017) 098 [arXiv: 1611.04603] [INSPIRE].

[37] A. Gimenez Grau, C. Kristjansen, M. Volk and M. Wilhelm, A Quantum Check of Non-Supersymmetric AdS/dCFT, JHEP 01 (2019) 007 [arXiv:1810.11463] [INSPIRE]. 
[38] A. Gimenez-Grau, C. Kristjansen, M. Volk and M. Wilhelm, A quantum framework for AdS/dCFT through fuzzy spherical harmonics on $S^{4}$, JHEP 04 (2020) 132 [arXiv: 1912.02468] [INSPIRE].

[39] L. Piroli, B. Pozsgay and E. Vernier, What is an integrable quench?, Nucl. Phys. B 925 (2017) 362 [arXiv : 1709. 04796] [InSPIRE].

[40] S. Ghoshal and A.B. Zamolodchikov, Boundary $S$ matrix and boundary state in two-dimensional integrable quantum field theory, Int. J. Mod. Phys. A 9 (1994) 3841 [Erratum ibid. 9 (1994) 4353] [hep-th/9306002] [INSPIRE].

[41] N. MacKay and V. Regelskis, Achiral boundaries and the twisted Yangian of the D5-brane, JHEP 08 (2011) 019 [arXiv: 1105.4128] [INSPIRE].

[42] O. Tsuchiya, Determinant formula for the six-vertex model with reflecting end, J. Math. Phys. 39 (1998) 135 [solv-int/9804010].

[43] M. Brockmann, J. De Nardis, B. Wouters and J.-S. Caux, A Gaudin-like determinant for overlaps of Néel and XXZ Bethe States, J. Phys. A 47 (2014) 145003 [arXiv:1401.2877].

[44] M. Brockmann, J. De Nardis, B. Wouters and J.-S. Caux, Néel-XXZ state overlaps: odd particle numbers and Lieb-Liniger scaling limit, J. Phys. A 47 (2014) 345003 [arXiv: 1403.7469].

[45] O. Foda and K. Zarembo, Overlaps of partial Néel states and Bethe states, J. Stat. Mech. 1602 (2016) 023107 [arXiv: 1512.02533] [INSPIRE].

[46] M. De Leeuw, T. Gombor, C. Kristjansen, G. Linardopoulos and B. Pozsgay, Spin Chain Overlaps and the Twisted Yangian, JHEP 01 (2020) 176 [arXiv:1912.09338] [INSPIRE].

[47] M. Fujita, W. Li, S. Ryu and T. Takayanagi, Fractional Quantum Hall Effect via Holography: Chern-Simons, Edge States, and Hierarchy, JHEP 06 (2009) 066 [arXiv: 0901.0924] [INSPIRE].

[48] M. de Leeuw, A.C. Ipsen, C. Kristjansen, K.E. Vardinghus and M. Wilhelm, Two-point functions in AdS/dCFT and the boundary conformal bootstrap equations, JHEP 08 (2017) 020 [arXiv: 1705. 03898] [INSPIRE].

[49] T. Gombor and B. Pozsgay, On factorized overlaps: Algebraic Bethe Ansatz, twists, and Separation of Variables, Nucl. Phys. B 967 (2021) 115390 [arXiv:2101.10354] [INSPIRE].

[50] C. Kristjansen, D. Müller and K. Zarembo, Duality relations for overlaps of integrable boundary states in AdS/dCFT, JHEP 09 (2021) 004 [arXiv:2106.08116] [INSPIRE].

[51] L. Frappat, P. Sorba and A. Sciarrino, Dictionary on Lie superalgebras, hep-th/9607161 [INSPIRE].

[52] Z. Tsuboi, Analytic Bethe Ansatz And Functional Equations Associated With Any Simple Root Systems Of The Lie Superalgebra sl $(r+1 \mid s+1)$, Physica A 252 (1998) 565 [arXiv:0911.5387] [INSPIRE].

[53] S.S. Gubser, I.R. Klebanov and A.M. Polyakov, Gauge theory correlators from noncritical string theory, Phys. Lett. B 428 (1998) 105 [hep-th/9802109] [INSPIRE].

[54] E. Witten, Anti-de Sitter space and holography, Adv. Theor. Math. Phys. 2 (1998) 253 [hep-th/9802150] [INSPIRE].

[55] B. Biran, A. Casher, F. Englert, M. Rooman and P. Spindel, The Fluctuating Seven Sphere in Eleven-dimensional Supergravity, Phys. Lett. B 134 (1984) 179 [INSPIRE]. 
[56] L. Castellani, R. D'Auria, P. Fré, K. Pilch and P. van Nieuwenhuizen, The Bosonic Mass Formula for Freund-rubin Solutions of $d=11$ Supergravity on General Coset Manifolds, Class. Quant. Grav. 1 (1984) 339 [InSPIRE].

[57] F. Bastianelli and R. Zucchini, Bosonic quadratic actions for $11-D$ supergravity on AdS(7/4) x S(4/7), Class. Quant. Grav. 16 (1999) 3673 [hep-th/9903161] [InSPIRE].

[58] S. Hirano, C. Kristjansen and D. Young, Giant Gravitons on $A d S_{4} \times \mathbb{C} P^{3}$ and their Holographic Three-point Functions, JHEP 07 (2012) 006 [arXiv:1205.1959] [INSPIRE].

[59] P. Yang, Y. Jiang, S. Komatsu and J.-B. Wu, D-branes and Orbit Average, arXiv:2103.16580 [INSPIRE].

[60] N. Drukker, J. Gomis and D. Young, Vortex Loop Operators, M2-branes and Holography, JHEP 03 (2009) 004 [arXiv:0810.4344] [INSPIRE].

[61] Y. Wang, Taming defects in $\mathcal{N}=4$ super-Yang-Mills, JHEP 08 (2020) 021 [arXiv: 2003.11016] [INSPIRE].

[62] M. Dedushenko and D. Gaiotto, Algebras, traces, and boundary correlators in $\mathcal{N}=4 S Y M$, JHEP 12 (2021) 050 [arXiv:2009.11197] [INSPIRE].

[63] M. Dedushenko and D. Gaiotto, Correlators on the wall and $\mathfrak{s l}_{n}$ spin chain, arXiv:2009.11198 [INSPIRE].

[64] G. Linardopoulos and K. Zarembo, String integrability of defect CFT and dynamical reflection matrices, JHEP 05 (2021) 203 [arXiv:2102.12381] [INSPIRE].

[65] P. Dorey, D. Fioravanti, C. Rim and R. Tateo, Integrable quantum field theory with boundaries: The Exact g function, Nucl. Phys. B 696 (2004) 445 [hep-th/0404014] [INSPIRE].

[66] Y. Jiang, S. Komatsu and E. Vescovi, Structure constants in $\mathcal{N}=4$ SYM at finite coupling as worldsheet g-function, JHEP 07 (2020) 037 [arXiv: 1906.07733] [INSPIRE].

[67] D. Binosi and L. Theussl, JaxoDraw: A Graphical user interface for drawing Feynman diagrams, Comput. Phys. Commun. 161 (2004) 76 [hep-ph/0309015] [INSPIRE].

[68] D. Binosi, J. Collins, C. Kaufhold and L. Theussl, JaxoDraw: A Graphical user interface for drawing Feynman diagrams. Version 2.0 release notes, Comput. Phys. Commun. 180 (2009) 1709 [arXiv:0811.4113] [INSPIRE]. 